\title{
Chemotherapy-Free Management of Follicular and Marginal Zone Lymphoma
}

\author{
Thomas A Ollila ${ }^{1,2}$ \\ Adam J Olszewski (D) ${ }^{1,2}$ \\ 'Department of Medicine, Alpert Medical \\ School of Brown University, Providence, \\ RI, USA; ${ }^{2}$ Division of Hematology- \\ Oncology, Rhode Island Hospital, \\ Providence, RI, USA
}

\begin{abstract}
Many patients with follicular (FL) or marginal zone lymphoma (MZL) are not eligible to receive immunochemotherapy due to advanced age or comorbidities. Recent innovations in the treatment of these indolent lymphomas provide options for multiple lines of chemotherapy-free management. More research is needed to determine which older patients are best served by a chemotherapy-free approach in the context of geriatric vulnerabilities. In the first line, regardless of disease burden, rituximab monotherapy can provide high rates of disease control with minimal toxicity, while judicious use of brief maintenance extends the duration of response. Radioimmunotherapy using ibritumomab tiuxetan is an effective and safe post-rituximab consolidation for older patients who have $<25 \%$ bone marrow involvement. The combination of rituximab and lenalidomide, although "chemotherapy-free", does not improve tolerability over immunochemotherapy. However, studies support lower doses and shorter duration of lenalidomide exposure as a means to improve safety without materially compromising efficacy for older individuals. Extranodal MZL can often be effectively controlled with low-dose radiation therapy, and splenic MZL has excellent outcomes with rituximab monotherapy. For many patients with relapsed FL/ MZL, simple retreatment with anti-CD20 antibodies will prove sufficient. Other currently available options for relapsed/refractory disease include ibritumomab tiuxetan, lenalidomide with rituximab, umbralisib as a potentially less toxic PI3K inhibitor, ibrutinib (for MZL), and tazemetostat (for FL, especially with EZH2 mutation). Emerging data with novel forms of immunotherapy (antibody-drug conjugates like polatuzumab vedotin or loncastuximab tesirine; T-cell-engaging bispecific antibodies like mosunetuzumab or epcoritamab; and chimeric antigen receptor CAR T-cells like axicabtagene ciloleucel) suggest that immune-directed approaches can produce very high and potentially durable responses in FL/MZL with limited toxicities, further obviating the need for chemotherapy.
\end{abstract}

Keywords: follicular lymphoma, marginal zone lymphoma, geriatric oncology, lenalidomide, ibritumomab tiuxetan, bispecific antibodies

\section{Introduction}

Indolent B-cell lymphomas (iBCL) are a group of slow-growing mature B-cell lymphomas that often affect older patients. In the United States (US), median age at diagnosis ranges from 65 for follicular lymphoma (FL), 67 for marginal zone lymphoma (MZL), 70 for chronic lymphocytic leukemia/small lymphocytic lymphoma (CLL/SLL), to 72 for lymphoplasmacytic lymphoma/Waldenström macroglobulinemia (LPL/WM). ${ }^{1}$ Many older patients with iBCL will have comorbid conditions and functional impairments that make treatment with cytotoxic chemotherapy difficult. ${ }^{2}$ Furthermore, advanced iBCL are not considered curable by standard therapy, yet average life expectancy is measured in years to decades and
Correspondence: Adam J Olszewski Department of Medicine, Alpert Medical School of Brown University, Rhode Island Hospital, 593 Eddy St., Providence, RI, 02903, USA

$\mathrm{Tel}+\mid$ 844-222-288|

Fax + 40I-444-89|8

Email adam_olszewski@brown.edu 
many patients will receive several lines of treatment. These factors highlight the importance of treatment choices that balance efficacy against toxicity considering patient's expected lifetime.

The 3 most common types of iBCL are FL (grade 1 to 3a), MZL, and SLL. Grade 3b FL is considered more aggressive and is managed using strategies appropriate for diffuse large B-cell lymphoma (DLBCL). MZL is further subclassified into nodal MZL (NMZL), splenic MZL (SMZL), and extranodal MZL (EMZL) of the mucosa-associated lymphoid tissue (MALT lymphoma). ${ }^{3}$ These subtypes have specific clinical features and management approaches. LPL/WM is uniquely characterized by complications of monoclonal IgM paraproteinemia, and its treatment follows specific patterns. ${ }^{4}$ In this review, we do not discuss CLL/SLL and LPL/WM, which follow disease-specific treatment pathways.

For the past two decades, the management of FL and MZL has relied on a few general principles. ${ }^{5,6}$ Early-stage disease (stage 1/2) could be treated with potentially curative intent using radiation therapy (or, in certain types of MZL, excision or antibiotic therapy). ${ }^{7-10}$ Asymptomatic patients with advanced stage disease would be typically observed without treatment or offered single-agent rituximab. ${ }^{11,12}$ Patients with a higher burden of disease would be mostly offered rituximab-containing immunochemotherapy. One current standard first-line immunochemotherapy approach is based on the Phase 3 German Study group indolent Lymphomas (StiL) trial, which showed improved progression-free survival (PFS) and fewer acute toxicities with rituximab plus bendamustine (BR) compared with the historical standard of R-CHOP (rituximab, cyclophosphamide, doxorubicin, vincristine, and prednisone). ${ }^{13}$ However, even BR is associated with $29 \%$ risk of grade $3 / 4$ neutropenia and $37 \%$ risk of infections. Furthermore, it leads to a prolonged T-cell depletion and potential opportunistic infections, including zoster, pneumonia, or CMV reactivations, particularly among older patients. ${ }^{14-16}$ Many older patients are thus understandably weary of such risks and prefer to avoid chemotherapy.

Recent advances allow older and unfit patients to expect reasonable efficacy with more tolerable, targeted therapies, which, if adequately sequenced, may obviate the need for any chemotherapy within their lifetime. In this review, we aim to present both established and novel chemotherapy-free approaches to systemic treatment for
FL and MZL, which are particularly (but not exclusively) relevant for older patients.

\section{Defining Candidates for Chemotherapy-Free Approach}

When treating an older patient with iBCL, clinicians face the complex question of whether the potential benefits of cytotoxic chemotherapy might be outweighed by risks. Geriatric assessment (GA) can help clarify this balance, although its use is less frequent in hematologic malignancies than in solid tumor oncology. ${ }^{17-19}$ Management of iBCL requires nuance to help decide if, when, and which therapy should be started, in the context of multiple options of variable intensity. The watch-and-wait approach can be applied to patients with asymptomatic and low-volume disease. ${ }^{11}$ The Groupe d'Etude des Lymphomes Folliculaires (GELF) or British National Lymphoma Investigation (BNLI) criteria provide objective definitions of high-burden FL. ${ }^{20,21}$ Prognostic scores like the FL International Prognostic Index (FLIPI) or MALT IPI may predict expected survival, but do not define an indication for treatment or a benefit from specific regimens. ${ }^{22-24}$ A dedicated GA can help guide the decision-making, providing insight into functional and nutritional status, polypharmacy, as well as psychosocial factors. GA has been used in diffuse large B-cell lymphoma (DLBCL) for selection of treatment intensity, but data in $\mathrm{BCL}$ are sparse. ${ }^{25,26}$ Observational studies have demonstrated that GA predicts shorter survival, treatment discontinuation, and the risk of hospitalization beyond simple "performance status" assessments, mostly in the context of cytotoxic chemotherapy. ${ }^{27}$ Clinical trials of chemotherapy-free approaches in indolent lymphomas have so far not used GA for patient selection. However, a recent Phase 2 study has demonstrated that patients age 65-80 who scored "fit" on the simplified comprehensive GA could be safely treated with a short course of immunochemotherapy (rituximab, bendamustine, plus mitoxantrone), achieving $78 \%$ rate of complete response (CR) and a 3 -year PFS of $67 \% .^{28}$ Only 4 of 72 participants discontinued chemotherapy for toxicity.

When comprehensive GA is not available, hematologists can assess patients' fitness using simple tools like the Timed Up and Go (TUG) test, which involves brisk walking back and forth for 3 meters from a sitting position. ${ }^{18}$ Needing $>30$ seconds to complete the TUG test correlates with significant frailty. In a prospective observational study of patients with hematologic malignancies (29\% various lymphomas), gait 
Table I Chemotherapy-Free Options for First-Line Treatment in FL or MZL

\begin{tabular}{|c|c|c|c|c|c|c|c|}
\hline Treatment & Disease & $\mathbf{N}$ & Age, Median (Range) & Gr. 3/4 AE & ORR & CR/CRu & Median PFS (y) \\
\hline \multicolumn{8}{|l|}{ Rituximab } \\
\hline $\mathbb{I R}^{32,33}$ & $\mathrm{FL}$ & 50 & $52(32-75)$ & $4 \%$ & $73 \%$ & $26 \%$ & 2.0 \\
\hline $\mathbb{I R}^{11}$ & $\mathrm{FL}$ & 84 & $60(33-86)$ & $6 \%$ & $77 \%$ & $47 \%$ & $>4^{\mathrm{a}}$ \\
\hline $\operatorname{IR}^{34,35}$ & FL/MZL & 321 & $57(29-82)$ & $7 \%^{b}$ & $74 \%^{b}$ & $41 \%^{b}$ & $1.45^{c}$ \\
\hline$M R^{\prime \prime}$ & $\mathrm{FL}$ & 192 & $60(27-87)$ & $11 \%$ & $88 \%$ & $69 \%$ & $>5^{a}$ \\
\hline$M R^{37}$ & $\mathrm{FL}$ & 270 & $55(25-82)$ & $9 \%$ & $63 \%$ & $16 \%$ & 7.4 \\
\hline $\mathrm{IR} \pm M \mathrm{R}^{38}$ & $\mathrm{FL}$ & 289 & $59(25-86)$ & $5 \%$ & $71 \%$ & $12 \%$ & $\sim 3^{a}$ IR, NR MR \\
\hline $\mathrm{IR} \pm \mathrm{MR}^{39}$ & MZL/SLL & 7I MZL & $66(30-86)$ & $6 \%$ & $52 \%$ (MZL) & I3\% (MZL) & I.4 IR, $4.8 \mathrm{MR}^{\mathrm{c}}$ \\
\hline $\operatorname{IR}^{69}$ & EMZL & 138 & $63(27-8 I)$ & $10 \%$ & $78 \%$ & $56 \%$ & 6.9 \\
\hline $\mathrm{IR} \pm M \mathrm{R}^{75}$ & SMZL & 108 & $65(4|-9|)$ & a & $92 \%$ & $65 \%$ & $>10$ \\
\hline \multicolumn{8}{|c|}{ Rituximab + lenalidomide } \\
\hline $\mathrm{R} 2^{52}$ & $\mathrm{FL}$ & 413 & 59 (30-89) & $65 \%$ & $61 \%$ & $48 \%$ & $77 \%$ at $3 y$ \\
\hline $\mathrm{R} 2^{57}$ & $\mathrm{FL}$ & 77 & $61(26-80)$ & $56 \%$ & $81 \%$ & $36 \%$ & 5.0 \\
\hline $\mathrm{R} 2^{56}$ & MZL & 30 & $58(36-77)$ & $\geq 33 \%^{a}$ & $93 \%$ & $70 \%$ & 5.0 \\
\hline \multicolumn{8}{|c|}{ Ibritumomab tiuxetan } \\
\hline${ }^{90} \mathrm{YIT}^{60}$ & $\mathrm{FL}$ & 50 & $60(37-81)$ & $\geq 30 \%^{a}$ & $94 \%$ & $86 \%$ & $63 \%$ at $3 y$ \\
\hline${ }^{90} \mathrm{YIT}^{61}$ & FL, MZL & 31 & $57(28-87)$ & $\geq 61 \%^{a}$ & $100 \%$ & $97 \%$ & $\sim 75 \%$ at $3 y^{a}$ \\
\hline${ }^{90} \mathrm{YIT}^{62}$ & $\mathrm{FL}$ & 59 & $66(51-83)$ & $48 \%$ & $87 \%$ & $56 \%$ & 2.2 \\
\hline${ }^{90} \mathrm{YIT}^{66}$ & $\mathrm{FL}$ & 74 & $61(28-80)$ & $56 \%$ & $96 \%$ & $69 \%$ & 3.4 \\
\hline${ }^{90} \mathrm{YIT}^{67}$ & MZL & 16 & $62(37-84)$ & $50 \%$ & $88 \%$ & $56 \%$ & 4.0 \\
\hline
\end{tabular}

Notes: ${ }^{a}$ Not explicitly reported; ${ }^{b}$ rituximab monotherapy arm; as reported in the MLI6865 trial; ${ }^{35}{ }^{c}$ time to treatment failure.

Abbreviations: AE, adverse event; CR/CRu, complete response/complete response unconfirmed; DOR, duration of response; EFS, event-free survival; EMZL, extranodal marginal zone lymphoma; IR, induction rituximab; MR, maintenance rituximab; NR, not reached; ORR, overall response rate; PFS, progression-free survival; R2, rituximab and lenalidomide; TTF, time to treatment failure; $y$, years; ${ }^{90} \mathrm{YIT}$, ibritumomab tiuxetan.

speed measured over a 4-meter walk strongly correlated with overall survival, unplanned hospitalizations, and emergency room visits - but did not appear to influence treatment intensity recommendation. ${ }^{29,30}$ Although clinical data on indolent lymphomas are lacking, in the CLL9 clinical trial of cytotoxic chemotherapy, the TUG test was a better predictor of survival than the assessment of comorbidities and of the instrumental activities of daily living. ${ }^{31}$ We recommend that clinicians formally assess their older patients with iBCL for functional capacity and that they consider chemotherapy-free regimens for frail individuals. On the one hand, age alone should not disqualify a fit older patient from receipt of chemotherapy, but on the other-patients with functional impairments and comorbidities may be the group benefitting most from treatment options that avoid cytotoxic chemotherapy.

\section{First-Line Therapy for FL and MZL}

Evidence for the use of chemotherapy-free treatments in iBCL is largely based on experience from FL, but some MZL-specific studies support their use for different MZL subtypes (Table 1). The principal options include singleagent rituximab, the combination of rituximab with lenalidomide, and radioimmunotherapy (Figure 1).

\section{Anti-CD20 Antibodies: Selective Use of Maintenance}

Rituximab is a chimeric monoclonal anti-CD20 antibody with over 20 years of cumulative experience in iBCL. First-line rituximab monotherapy in low-burden FL results in an overall response rate (ORR) of $73 \%$, a rate of complete response (CR) or complete response unconfirmed $(\mathrm{CRu})$ of $26 \%$ at the end of therapy which deepens to $52 \%$ over time, median PFS of 24 months, and median duration of response (DOR) of 29 months. ${ }^{32,33}$ The disease control is also excellent for symptomatic patients: in a long-term follow-up of 2 Nordic Lymphoma Group trials of rituximab ( \pm interferon alpha) in symptomatic iBCL ( $84 \%$ FL), $36 \%$ of patients never needed chemotherapy, and OS at 10 years was $75 \%$ - similar to trials that used first-line immunochemotherapy. ${ }^{34,35}$ One international randomized study compared watchful waiting with rituximab 


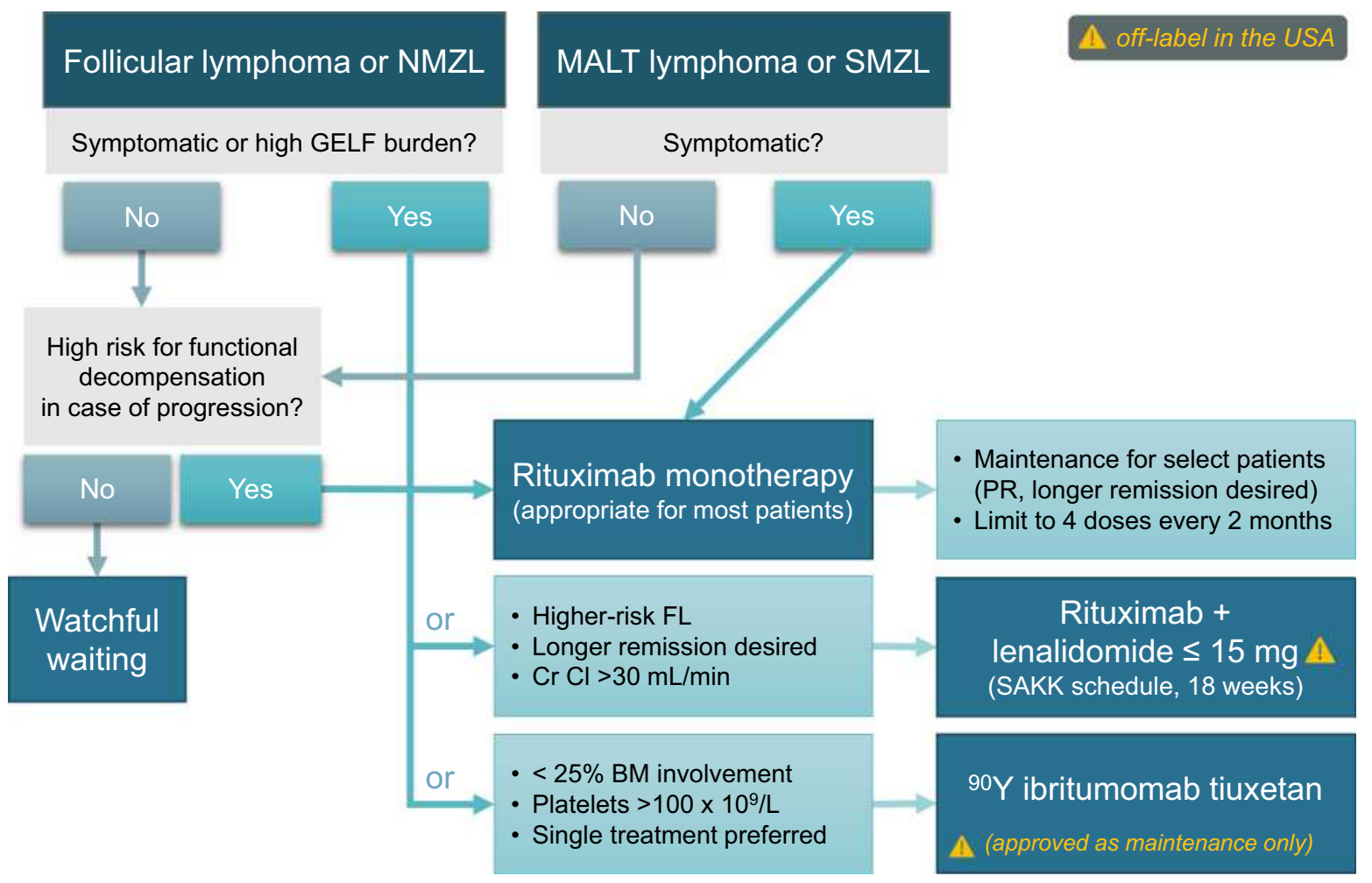

Figure I Chemotherapy-free options for first-line systemic therapy of FL or MZL (note that some options are off-label in the USA).

Abbreviations: BM, bone marrow; $\mathrm{CrCl}$, creatinine clearance; FL, follicular lymphoma; GELF, Groupe d-Etude des Lymphomes Folliculaires; MALT, mucosa-associated lymphoid tissue; NMZL, nodal marginal zone lymphoma; PR, partial response; SAKK, Swiss Group for Clinical Cancer Research; SMZL, splenic marginal zone lymphoma.

\pm 2-year maintenance in low-burden FL. ${ }^{11}$ Following a single 4-week course of rituximab, $78 \%$ of participants did not require any therapy for over 3 years; this proportion was even higher (88\%) if maintenance was applied. The overall quality of life was similar among patients undergoing watchful waiting versus rituximab therapy, except for improved measures of mental adjustment to cancer and illness coping with active therapy. ${ }^{11}$ In a phase 2 trial by the Swiss Group for Clinical Cancer Research (SAKK) which enrolled patients regardless of disease burden or prior chemotherapy, a brief (4 doses every 2 months) rituximab maintenance also extended median event-free survival (EFS) to 24 months compared with 13 months with rituximab induction alone. ${ }^{36}$ However, longer maintenance (up to 5 years) did not provide additional benefit. ${ }^{37}$ Similarly, the E4402 trial randomized patients with $\mathrm{FL}$ or other $\mathrm{BBCL}$ (including MZL) to maintenance rituximab or simple retreatment in case of progression, showing no significant difference in median time to treatment failure (4.3 versus 3.9 years for FL, $P=0.54$ ) or quality of life. ${ }^{38-40}$ Therefore, the potential benefits of maintenance rituximab should be balanced against the burden of extended therapy and associated infectious toxicity, particularly for patients with other risk factors for infections.

The subcutaneous formulation of rituximab with hyaluronidase may provide similar efficacy with increased convenience, although it is not currently approved in the first-line setting as monotherapy. ${ }^{41}$ Given the established efficacy of the formulation, it can be considered after at least 1 intravenous dose of rituximab is given without a severe infusion reaction. Recent data also support the use of rituximab biosimilars for first-line therapy. ${ }^{42}$ Of note, another anti-CD20 antibody ofatumumab failed to show improvement in outcomes over rituximab. ${ }^{43,44}$

Obinutuzumab is a type II glycoengineered anti-CD20 IgG monoclonal antibody with increased direct cytotoxic effect as well as antibody-dependent cellular cytotoxicity. ${ }^{45}$ It has shown efficacy for iBCL in both relapsed/refractory and first-line settings, extends PFS when combined with chemotherapy instead of rituximab, and has gained FDA approval in combination with chemotherapy for treatment of FL. ${ }^{4-49}$ However, when used as monotherapy in the phase 2 randomized GAUSS trial in FL, obinutuzumab increased ORR without any PFS or toxicity advantage over rituximab. ${ }^{47}$ Therefore, we recommend rituximab rather 
than obinutuzumab for first-line monotherapy in $\mathrm{BCL}$, with a selective use of maintenance for responding patients who value a longer remission over potential retreatment.

\section{Lenalidomide and Rituximab: Lower Dose, Shorter Duration}

Lenalidomide in combination with rituximab (R2) has shown high activity in FL and MZL and is currently FDAapproved for these histologies after at least 1 line of therapy. In previously untreated patients, phase 2 studies showed ORR exceeding $90 \%$ and CR rates exceeding $70 \% .^{50,51}$ The subsequent phase 3 RELEVANCE trial compared R2 with chemoimmunotherapy (RCHOP in $72 \%$ ) in untreated FL. ${ }^{52}$ Lenalidomide was dosed at $20 \mathrm{mg}$ daily on days 1-21 for 6 four-week cycles, and then decreased to $10 \mathrm{mg}$ for additional 12 months in patients with $\mathrm{CR} / \mathrm{CRu}$, for a total of 18 months of therapy. Rituximab was given weekly during cycle 1 , monthly for cycles 2-6, and every 8 weeks for additional 12 cycles. However, the overall toxicity profile was not lower than with immunochemotherapy. In fact, more patients receiving R2 had dose reductions, interruptions, or early discontinuations, and $67 \%$ experienced grade $3 / 4$ adverse events which included neutropenia in $32 \%$ and rash/cutaneous reactions in 7\%. Less severe, but important (considering prolonged exposure) toxicities included anemia (66\%), diarrhea (37\%), fatigue (23\%), nausea $(20 \%)$, abdominal pain $(15 \%)$ and myalgias (14\%). Although late or longitudinal toxicities are insufficiently evaluated in clinical trials, experience with lenalidomide in FL from the Alliance 50401 trial suggests that fatigue in lenalidomide decreases over time, but neutropenia is cumulative and steady. ${ }^{53}$ No excess in secondary cancers was reported in the RELEVANCE trial. ${ }^{52}$ With no significant advantage over chemotherapy in PFS (HR, 1.10; 95\% CI 0.85-1.43), other efficacy or toxicity endpoints, and the need for 1.5 years of continuous therapy, the R2 regimen, while technically "chemotherapy-free," cannot be considered an improvement for older/unfit patients with FL. It also could not be safely combined with a third targeted agent like ibrutinib or idelalisib. ${ }^{54,55}$ In MZL, R2 retains a high efficacy with reported ORR of $93 \%$, CR of $70 \%$, and median PFS of 60 months, but the toxicity profile is similar to FL. 56

Importantly, the immunomodulatory effects of lenalidomide can be achieved with lower doses of the drug. The SAKK 35/10 phase 2 trial randomized 154 previously untreated FL patients to two 4-week courses of rituximab given 3 months apart, with or without lenalidomide $15 \mathrm{mg}$ administered continuously for 18 weeks. ${ }^{57}$ Lenalidomide was started 2 weeks before the first infusion of rituximab and further dose-reduced to $5 \mathrm{mg}$ for creatinine clearance $<60 \mathrm{~mL} / \mathrm{min}$. The ORR to this $\mathrm{R} 2$ version was $81 \%$ and the $\mathrm{CR} / \mathrm{CRu}$ was $36 \%$ (61\% by independent review), with median PFS of 5 years - all better than for rituximab alone. Grade $\geq 3$ toxicity was higher with lenalidomide (56\% versus $22 \%$ ), but grade $3 / 4$ neutropenia $(23 \%)$ appeared less frequent than in the RELEVANCE trial and no other grade $3 / 4$ toxicity occurred in more than $5 \%$. Common lower-grade adverse events included fatigue $(52 \%)$, infections $(30 \%)$, rash $(27 \%)$, diarrhea $(25 \%)$, cough $(25 \%)$, and nausea/vomiting (22\%). These results suggest that addition of lenalidomide to rituximab can provide a benefit for older patients who need higher ORR or longer duration of remission, but lenalidomide can be administered at lower doses and for a short duration. The immunomodulatory effects of lenalidomide among older patients need to be investigated in further research considering immune senescence. It is important to note that lenalidomide increases the risk of thromboembolism and thromboprophylaxis should be considered.

\section{Radioimmunoconjugates}

Although maintenance rituximab can extend PFS after the initial induction, an alternative, albeit underused, approach involves consolidation using the ${ }^{90} \mathrm{Y}$ radioimmunoconjugate ibritumomab tiuxetan $\left({ }^{90}\right.$ YIT). ${ }^{90}$ YIT was initially studied after cytotoxic chemotherapy, ${ }^{58,59}$ but it has also been applied as a single-agent for early- and advanced-stage iBCL. ${ }^{60-62}{ }^{90}$ YIT has the potential advantage of delivery as a single course completed within 10 days, which includes the 2 rituximab doses required 7-9 days before and on the day of ${ }^{90}$ YIT infusion. Because of the potential for toxicity resulting from bone marrow irradiation, ${ }^{90} \mathrm{YIT}$ can only be applied to patients with a platelet count $>100 \times 10^{9}$ / $\mathrm{L}$ and less than $25 \%$ lymphomatous infiltration of the bone marrow. Concerns about secondary myeloid malignancy often relegate the use of ${ }^{90}$ YIT to subsequent lines of therapy, but the risk is actually not increased when ${ }^{90} \mathrm{YIT}$ is given before any exposure to cytotoxic chemotherapy. ${ }^{60,63-65}$ In phase 2 studies, ORR in FL was $87-94 \%$, CR rate was $56-86 \%$, PFS at 3 years was $58-63 \%$, and overall survival was $>90 \% .^{60,62,66}$ Grade 3/4 non-hematologic toxicities were exceptionally rare, 
although neutropenia or thrombocytopenia were noted in up to $30 \%$, with nadir at $\sim 35$ days and universal resolution after 14 weeks from treatment. ${ }^{90} \mathrm{YIT}$ is equally effective in MZL, but it is only approved for relapsed/refractory $\mathrm{iBCL}$ or as consolidation in FL after first-line chemotherapy. ${ }^{67}$

${ }^{90}$ YIT provides an attractive single-course therapy for iBCL for older patients given its efficacy, relatively mild non-hematologic toxicity and low burden of treatment visits, but it requires coordination between the hematologist and the nuclear medicine department and is not universally available. In addition, the requirement for baseline bone marrow assessment and for monitoring of the extended myelosuppression may pose barriers for some older individuals. ${ }^{90}$ YIT has not been studied in SMZL, which typically extensively involves the bone marrow, and it should be used with caution in this disease.

\section{Special Cases: SMZL and MALT Lymphoma}

While immunochemotherapy is typically applied to $\mathrm{FL}$ and NMZL, rituximab monotherapy is often sufficient for advanced MALT lymphoma or SMZL regardless of age, with no detriment to survival and with less toxicity when chemotherapy is omitted., ${ }^{2,71}$ In the phase 3 study (IELSG-19) comparing first-line systemic chlorambucil, rituximab, or both drugs in MALT lymphoma, singleagent rituximab has shown ORR of $78 \%$, CR rate of $56 \%$, and median event-free survival (EFS) of 5.6 years. ${ }^{69}$ An important consideration for older and unfit patients is the high radiosensitivity of MALT lymphoma, which can often be effectively controlled with minimal doses (4 Grays) of radiation therapy delivered to single or multiple sites of disease. ${ }^{72}$

Rituximab is also an excellent first-line option for SMZL obviating the use of splenectomy for most patients. ${ }^{71,73,74}$ In fact, observational data suggest no benefit of the addition of chemotherapy to the monoclonal antibody in this disease for older individuals. ${ }^{70}$ The largest phase 2 trial used a 6-week rituximab induction followed by 1-2 years of maintenance. It reported ORR of $92 \%$, $\mathrm{CR} / \mathrm{CRu}$ of $65 \%$, and 5-year PFS of $71 \%{ }^{75}$ Patients with SMZL should always be screened for the presence of hepatitis $\mathrm{C}$, as regression of the lymphoma has been noted after treatment for this infection. ${ }^{76,77}$ For patients unsuitable for any systemic therapy or splenectomy, radiation is a seldom used but efficacious option. ${ }^{78}$

\section{Relapsed/Refractory Disease}

Among patients who experience relapsed or refractory FL/ MZL after initial therapy, the extent of symptoms and disease burden, duration of first response ( $<$ or $\geq 2$ years), as well as preference for intravenous or orally administered therapy will determine further management (Figure 2). ${ }^{79,80}$ Clinical trials are always a consideration in this setting and should be offered to eligible older and less fit patients. Many patients with $\mathrm{iBCL}$ experience asymptomatic or slow progression which might not require immediate therapythough, as in the first-line setting, the risk of decompensation during watchful waiting should be considered in the context of comorbidities, psychosocial support, tumor location and bulk. Rapidly progressive recurrence should prompt evaluation of a histologic transformation. Clinicians should also remember that low-intensity chemotherapy (for example, chlorambucil or judiciously dosed $\mathrm{BR}$ ) can control $\mathrm{FL}$ or MZL for many patients with toxicity that is no worse, and sometimes better, than some "chemotherapy-free" approaches. $^{21,69,81-83}$

\section{Retreatment with Rituximab}

Patients initially treated with single-agent rituximab and not on maintenance can be often simply retreated upon progression according to the RESORT trial paradigm. ${ }^{38}$ In a phase 2 trial, retreatment with rituximab (at median 14.5 months from the previous course) resulted in ORR of $40 \%$, including $11 \%$ $\mathrm{CR}$, and an estimated median time to progression of 18 months. ${ }^{84}$ Using an alternative antibody (ofatumumab or obinutuzumab) does not appear to meaningfully improve outcomes (Table 2) ${ }^{43,47}$ However, for patients who experience a rituximab-refractory FL/MZL or a symptomatic relapse within 2 years of prior therapy, we favor an alternative approach when life expectancy exceeds 2 more years because a second remission with rituximab alone is likely to be brief.

\section{Radioimmunoconjugates}

The use of ${ }^{90}$ YIT in relapsed iBCL is FDA-approved and supported by phase 2 and 3 trials. ${ }^{85-87}$ Among patients with rituximab-refractory FL (defined as no response or progression within 6 months), ORR to ${ }^{90} \mathrm{YIT}$ is $74 \%$, CR rate is $15 \%$, and median time to progression is 7 months. ${ }^{88}$ In a subsequent phase 3 trial, ORR was $80 \%$, CR rate was $30 \%$, and median time to progression was 11 months. ${ }^{89} \mathrm{In}$ relapsed/refractory MALT lymphoma, this approach resulted with ORR of $90-94 \%$, CR rate of $62-77 \%$, and median PFS exceeding 3 years. ${ }^{85,90}$ Therefore, ${ }^{90}$ YIT may 


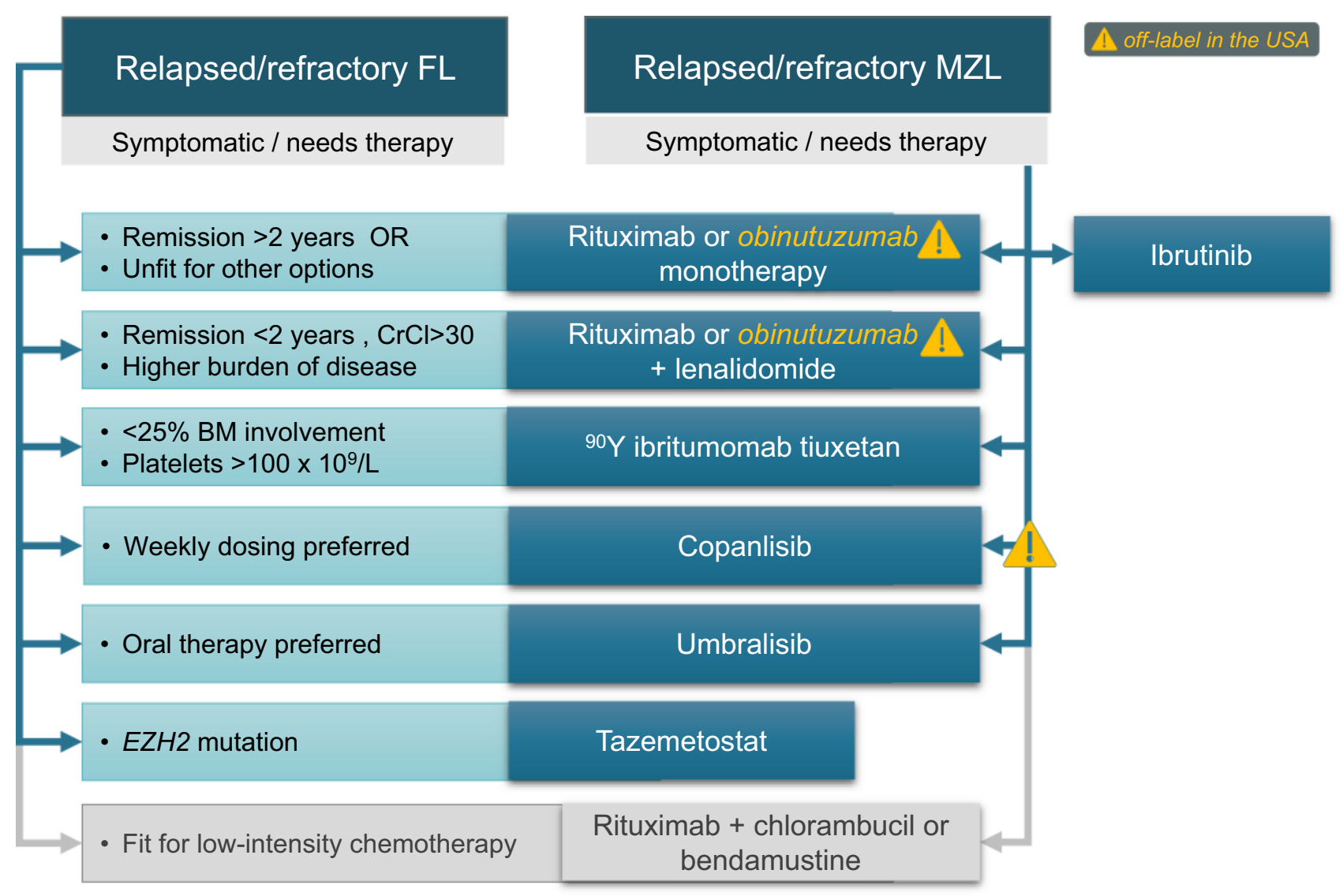

Figure 2 FDA-approved chemotherapy-free options for relapsed or refractory FL or MZL (note that some options are off-label in the USA for either one or both histologies).

Abbreviations: $\mathrm{BM}$, bone marrow; $\mathrm{CrCl}$, creatinine clearance; $\mathrm{FL}$, follicular lymphoma; IV, intravenous; MZL, marginal zone lymphoma.

be an excellent option for an older patient with a relapsing FL/MZL and no prior exposure to chemotherapy.

\section{Lenalidomide \pm Rituximab or Obinutuzumab}

Lenalidomide plus an anti-CD20 antibody offers an attractive approach for patients who can tolerate the associated toxicity, who did not receive lenalidomide as first-line therapy, and who have creatinine clearance $>30 \mathrm{~mL} / \mathrm{min}$. Similar to the first-line setting, this option is comparable in efficacy and toxicity to cytotoxic chemotherapy and may not be suitable for frail individuals who would otherwise not accept or withstand chemotherapy.

R2 is approved in both FL and MZL based on the phase 3 AUGMENT study which showed longer PFS (39 versus 14 months) and higher ORR (78\% versus 53\%) compared with rituximab. ${ }^{91}$ The median age of participants was 64 (range, 26-86), and 84\% had prior rituximab exposure. Toxicity was unfortunately also higher with R2: $69 \%$ of patients experienced grade $3 / 4$ toxicities (primarily hematologic) and $26 \%$ had at least 1 serious adverse event. Neutropenia was the most common adverse event (58\%), responsive to dose interruptions and reductions of lenalidomide which occurred in $64 \%$ and $26 \%$ of participants, respectively. It is important to note that the lenalidomide dose was $20 \mathrm{mg}$ for patients with creatinine clearance $>60 \mathrm{~mL} / \mathrm{min}$, but only $10 \mathrm{mg}$ for those with clearance $30-59 \mathrm{~mL} / \mathrm{min}$. We suggest aggressive dose adjustments (including a potential start at $50 \%$ of the target dose with subsequent escalation) for older/unfit patients to avert the risk of toxicity-related hospitalizations and early discontinuations. In the phase 2 GALEN study for relapsed/refractory FL, lenalidomide with obinutuzumab also provided a high ORR (79\%) and 2-year PFS (65\%). ${ }^{92}$ However, adverse events were common with asthenia in $61 \%$, neutropenia in $43 \%$, diarrhea in $40 \%$, bronchitis in $41 \%$, and muscle spasms in $39 \%$. While the advantage of obinutuzumab in this setting remains uncertain, responses may be higher for patients who had short ( $<2$ years) remissions to prior rituximab-based therapy. 
Table 2 Chemotherapy-Free Options for Relapsed/Refractory FL or MZL

\begin{tabular}{|c|c|c|c|c|c|c|c|c|c|}
\hline Treatment & $\mathbf{N}$ & N FL & $\begin{array}{c}\mathbf{N} \\
\text { MZL }\end{array}$ & $\begin{array}{l}\text { Age, Median } \\
\text { (Range) }\end{array}$ & $\begin{array}{l}\text { Prior Lines, Median } \\
\text { (Range) }\end{array}$ & $\begin{array}{c}\text { Gr 3/4 Adverse } \\
\text { Events }\end{array}$ & ORR & $\begin{array}{l}\text { CR/ } \\
\text { CRu }\end{array}$ & $\begin{array}{c}\text { Median } \\
\text { PFS } \\
(\mathrm{mo})\end{array}$ \\
\hline \multicolumn{10}{|l|}{ Anti-CD20 monotherapy } \\
\hline Obinutuzumab ${ }^{46}$ & 40 & 34 & & $61(42-79)$ & $3(1-11)$ & $33 \%$ & $55 \%$ & $9 \%$ & 12 \\
\hline Rituximab $^{47}$ & & 75 & 5 & $60(38-80)$ & $2(1-6)$ & $15 \%$ & $33 \%$ & $5 \%$ & 25 \\
\hline Obinutuzumab ${ }^{47}$ & & 74 & 6 & $62(33-84)$ & $2(1-7)$ & $15 \%$ & $45 \%$ & $12 \%$ & 18 \\
\hline Rituximab $^{43}$ & 219 & 214 & & $62(26-85)$ & $2(I-9)$ & $28 \%$ & $66 \%$ & $20 \%$ & 19 \\
\hline Ofatumumab ${ }^{43}$ & 219 & 214 & & 61 (27-90) & $2(1-10)$ & $37 \%$ & $50 \%$ & $16 \%$ & 16 \\
\hline Rituximab $^{89}$ & 70 & 58 & & $57(36-78)$ & $2(1-5)$ & a & $56 \%$ & $20 \%$ & $10^{b}$ \\
\hline \multicolumn{10}{|l|}{ Ibritumomab tiuxetan } \\
\hline${ }^{90} \mathrm{YIT}^{89}$ & 73 & 55 & & $60(29-80)$ & $2(1-6)$ & $\geq 60 \%^{\mathrm{a}}$ & $80 \%$ & $34 \%$ & $11^{b}$ \\
\hline${ }^{90} \mathrm{YIT}^{85}$ & 30 & & 30 & $57(36-83)$ & 2 & $\geq 60 \%^{\mathrm{a}}$ & $90 \%$ & $77 \%$ & NR \\
\hline${ }^{90} \mathrm{YIT}^{90}$ & 16 & & 16 & $58(19-77)$ & 2 & $\geq 44 \%^{\mathrm{a}}$ & $94 \%$ & $63 \%$ & 38 \\
\hline \multicolumn{10}{|l|}{$\begin{array}{l}\text { Lenalidomide } \pm \text { anti-CD20 } \\
\text { antibody }\end{array}$} \\
\hline Lenalidomide $^{93}$ & 45 & 45 & & $63(34-85)$ & NR & $58 \%$ & $53 \%$ & $20 \%$ & $13^{b}$ \\
\hline Lenalidomide $^{94}$ & 43 & 22 & 3 & 63 (42-89) & $3(1-17)$ & NS & $23 \%$ & $5 \%$ & 4 \\
\hline Lenalidomide + rituximab ${ }^{91}$ & 178 & 147 & 31 & $64(26-86)$ & $I(I-12)$ & $69 \%$ & $78 \%$ & $34 \%$ & 39 \\
\hline 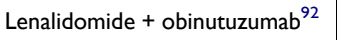 & 88 & 88 & & 64 (39-87) & $2(I-7)$ & $\geq 44 \%$ & $79 \%$ & $39 \%$ & $65 \%$ at $2 y$ \\
\hline \multicolumn{10}{|l|}{ PI3K inhibitors } \\
\hline Umbralisib $^{103}$ & 208 & 117 & 69 & $66(29-88)$ & $2(1-10)$ & $53 \%$ & $\begin{array}{c}\mathrm{FL} \\
45 \% \\
\mathrm{MZL} \\
49 \%\end{array}$ & $\begin{array}{c}\text { FL 5\% } \\
\text { MZL } \\
16 \%\end{array}$ & $\begin{array}{l}\text { FL: II } \\
\text { MZL: NR }\end{array}$ \\
\hline Copanlisib ${ }^{104,105}$ & 142 & 104 & 23 & $63(25-82)$ & $3(2-9)$ & $\geq 53 \%^{\mathrm{a}}$ & $\begin{array}{c}\mathrm{FL} \\
59 \% \\
\mathrm{MZL} \\
70 \%\end{array}$ & $\begin{array}{c}\mathrm{FL} \\
14 \% \\
\mathrm{MZL} \\
9 \%\end{array}$ & 11 \\
\hline Idelalisib $^{98}$ & 125 & 72 & 15 & $64(33-87)$ & $4(2-12)$ & $54 \%$ & $\begin{array}{c}\mathrm{FL} \\
54 \% \\
\mathrm{MZL} \\
47 \%\end{array}$ & $6 \%$ & 11 \\
\hline Duvelisib ${ }^{101}$ & 129 & 83 & 18 & $65(30-90)$ & $3(1-18)$ & $88 \%$ & $\begin{array}{c}\mathrm{FL} \\
42 \% \\
\mathrm{MZL} \\
39 \%\end{array}$ & $\begin{array}{c}\mathrm{FL} \mathrm{I \%} \\
\mathrm{MZL} \\
6 \%\end{array}$ & 9 \\
\hline \multicolumn{10}{|l|}{ BTK inhibitor } \\
\hline Ibrutinib 106 & 110 & 110 & & $62(28-87)$ & $3(2-13)$ & $48 \%$ & $21 \%$ & $11 \%$ & 5 \\
\hline Ibrutinib 107 & 40 & 40 & & $64(46-82)$ & $3(1-11)$ & $43 \%$ & $38 \%$ & $13 \%$ & 14 \\
\hline Ibrutinib 109 & 63 & & 63 & $66(30-92)$ & $2(I-9)$ & $71 \%$ & $58 \%$ & $3 \%$ & 16 \\
\hline \multicolumn{10}{|l|}{ EZH2 inhibitor } \\
\hline $\begin{array}{l}\text { Tazemetostat }{ }^{\prime \prime \prime} \\
\left(E Z H 2^{\text {mut }}\right)\end{array}$ & 45 & 45 & & $62(57-68)$ & $2(2-43)$ & $27 \%$ & $69 \%$ & $13 \%$ & 14 \\
\hline $\begin{array}{l}\text { Tazemetostat } \\
\left(E Z H 2^{w t}\right)\end{array}$ & 54 & 54 & & $61(53-67)$ & $3(2-5)$ & $27 \%$ & $35 \%$ & $4 \%$ & 11 \\
\hline
\end{tabular}

Notes: ${ }^{a}$ Not explicitly stated; 'time to progression; 'serious adverse events.

Abbreviations: AE, adverse event; ORR, overall response rate; CR/CRu, complete response/complete response unconfirmed; PFS, progression-free survival; mo, months; NR, not reached; $y$, years; ${ }^{90} \mathrm{YIT}$, ibritumomab tiuxetan. 
Lenalidomide (started at $15 \mathrm{mg}$ during cycle 1 , and escalated to $20 \mathrm{mg}$ in cycles 2 to 12 ) has also been studied as a single agent, providing $53 \%$ ORR and $20 \%$ CR rate in recurrent $\mathrm{FL}$ with median time to progression of 1.1 year. $^{93}$ These results were inferior, and toxicity not lower, compared with R2, suggesting an important synergy with rituximab, which is consistent with the immunomodulatory mechanism of action for lenalidomide. ORR was even lower $(23 \%)$ in a study enrolling various iBCL. ${ }^{94}$ In the AUGMENT trial, no difference in the global quality of life assessment between $\mathrm{R} 2$ and lenalidomide was reported at any timepoint. ${ }^{95}$ Therefore, we recommend using lenalidomide with an anti-CD20 antibody whenever possible. For elderly patients who find frequent visits to an infusion center burdensome and desire all-oral regimens, we favor other options discussed below.

\section{New PI3K Inhibitors: More Specificity, Less Toxicity}

The phosphatidylinositol 3-kinase (PI3K) inhibitors target the deregulated PI3K pathway important for B-cell receptor signaling, cellular proliferation, and anti-tumor immunity. ${ }^{96}$ Currently, four PI3K inhibitors agents are approved by the FDA: one intravenous (copanlisib, approved for FL) and targeting predominantly $\mathrm{PI} 3 \mathrm{~K} \alpha / \delta$, and three orally administered PI $3 \mathrm{~K} \delta \pm \gamma$ inhibitors: idelalisib (FL, SLL/CLL), duvelisib (FL, SLL/CLL), and umbralisib (FL, MZL). Many clinicians find this class of drugs challenging because of differences between individual agents and because of severe infectious and autoimmune toxicity associated with some. ${ }^{97}$ However, the novel PI3K $\delta$ inhibitor umbralisib appears to have improved toxicity, restoring an important lower-intensity option for older patients with FL or MZL.

Idelalisib was the first approved PI3K $\delta$ inhibitor based on a phase 2 trial that reported a $57 \%$ ORR in relapsed/refractory iBCL with median PFS of 11 months. ${ }^{98}$ Toxicities included neutropenia (56\%, grade $\geq 3$ in $27 \%$ ), diarrhea ( $43 \%$, grade $\geq 3$ in $13 \%$ ), fatigue ( $30 \%$, grade $\geq 3$ in $2 \%$ ), nausea $(30 \%$, grade $\geq 3$ in $2 \%)$, cough $(29 \%$, no grade $\geq 3)$ and pyrexia ( $28 \%$, grade $\geq 3$ in $2 \%$ ), as well as $13 \%$ grade $3 / 4$ hepatitis. Serious toxicities were even more frequent in a real-world sample of older Medicare beneficiaries receiving idelalisib. ${ }^{99}$ Longer use has amplified the safety concerns about colitis, hepatitis, pneumonitis, neutropenia, and opportunistic infections (CMV, Pneumocystis jirovecii pneumonia) and ultimately dissuaded many clinicians from routine use of idelalisib. ${ }^{100}$ Duvelisib was also studied in a phase 2 study of iBCL patients after median 3 lines of therapy. ORR was $47 \%$ and median PFS 9.5 months. ${ }^{101}$ Adverse effects were similar to idelalisib, including diarrhea ( $49 \%$, grade $\geq 3$ in $15 \%$ ), neutropenia ( $29 \%$, grade $\geq 3$ in $25 \%$ ), nausea ( $29 \%$, grade $\geq 3$ in $2 \%$ ), fatigue ( $28 \%$, grade $\geq 3$ in $5 \%$ ) and cough $(27 \%$, no grade $\geq 3$ ). Grade 3 colitis and pneumonitis were seen in $8 \%$ and $5 \%$, respectively, and a third of patients discontinued duvelisib because of toxicity.

Umbralisib is a novel drug in this class, characterized by substantially improved PI3K $\delta$ specificity and additional anti-

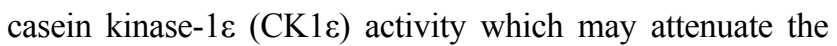
autoimmune toxicities. ${ }^{102,103}$ It is also the only oral PI3K inhibitor dosed once daily ( $800 \mathrm{mg}$ ), and has gained FDA approval for relapsed/refractory MZL and FL. In the registration phase 2 trial of 208 patients with iBCL ( $56 \%$ FL, 33\% MZL) after median 2 prior therapies, the ORR was $45 \%$ in FL ( $5 \%$ CR) and 49\% in MZL ( $16 \%$ CR), while median PFS was 11 months and not reached, respectively. ${ }^{103}$ Rates of grade $3 / 4$ diarrhea (10\%), hepatitis (7\%) and neutropenia (11\%) appeared lower than with other PI3K inhibitors, and colitis and pneumonitis were very rare (any grade events: $1.9 \%$ and $1.4 \%$, respectively). Considering this improved toxicity profile and convenience of once-daily dosing, umbralisib may be the PI3K inhibitor of choice for older or more frail patients. However, prophylaxis against Pneumocystis jirovecii and zoster, monitoring of CMV viremia, as well as strict dose reductions or holds are mandatory to maintain safety with this drug.

Copanlisib predominantly targets $\mathrm{PI} 3 \mathrm{~K} \alpha$ and $\mathrm{PI} 3 \mathrm{~K} \delta$ isoforms, resulting in a unique adverse event profile with transient hypertension, hyperglycemia, or neutropenia, but few long-term immune toxicities typical of $\mathrm{PI} 3 \mathrm{~K} \delta / \gamma$ targeting agents. It is approved for FL after $\geq 2$ prior systemic therapies and has a breakthrough designation for MZL. ${ }^{104,105}$ In the phase 2 registration trial, the ORR in FL was 59\%, CR rate was 14\%, and median PFS was 11 months. ${ }^{104}$ In MZL, copanlisib yielded ORR of $78 \%$ with median PFS of 24 months; CR (13\%) occurred in SMZL. ${ }^{105}$ Toxicity was substantial, including any grade fatigue in $52 \%$, diarrhea in $48 \%$, hyperglycemia in $48 \%$ (grade $3 / 4$ in 39\%), and hypertension in $44 \%$ (grade $3 / 4$ in $39 \%$ ), with $74 \%$ of patients requiring dose delays. Given the availability of oral alternatives, the use of copanlisib makes the most sense in the settings of financial barrier to accessing oral agents, or for patients who prefer weekly dosing. 


\section{Ibrutinib: For MZL Only}

Although best known for their use in SLL/CLL and mantle cell lymphoma, Bruton tyrosine kinase (BTK) inhibitors have been evaluated in both FL and MZL. ${ }^{106-108}$ The efficacy in FL is notably low: in a larger phase 2 trial, the ORR was only $21 \%$ with median PFS of 5 months, though in a smaller study it reached $38 \% .{ }^{106,107}$ In the latter experience, FL with CARD11 mutations was resistant to ibrutinib, while duration of response was longer with KMT2D and FOXO1 mutations. ${ }^{107}$ If next-generation sequencing becomes routinely applied in relapsed/refractory FL, knowledge of CARD11, KMT2D, and FOXO1 mutation status may allow a more personalized use of ibrutinib in FL. At present, single-agent BTK inhibitors in FL do not indicate sufficient efficacy.

In MZL, the use of ibrutinib is more compelling, as it attains 58\% ORR ( $81 \%$ for those pre-treated with rituximab only) with a median PFS of 16 months and median duration of response of 28 months. ${ }^{108,109}$ It is now FDAapproved for use in relapsed/refractory MZL. Ibrutinib is tolerable among older patients, with the oldest patient enrolled in a phase 2 trial being 92 years old. Grade $\geq 3$ AEs occurred in $71 \%$ of patients including grade $\geq 3$ infection in $22 \% .{ }^{109}$ Most adverse events were grade $1 / 2$, and grade $\geq 3$ events included also anemia (16\%), pneumonia $(8 \%)$ and fatigue $(6 \%)$. Ibrutinib is thus an excellent option for older patients whose MZL is refractory to rituximab monotherapy. Clinicians should be mindful of its potential cardiovascular toxicity (hypertension, atrial fibrillation), as well as interactions with anticoagulants and certain medications common in the elderly (diltiazem, verapamil). Ongoing studies explore the activity of other BTK inhibitors in MZL.

\section{Tazemetostat: Personalized Therapy for FL with EZH2 Mutation}

Tazemetostat, an oral inhibitor of the enhancer of zeste homolog 2 (EZH2), has generated significant interest in FL, where the prevalence of activating EZH2 mutations reaches $22 \% .{ }^{110}$ Tazemetostat is approved in the US for FL with EZH2 mutation, or for any FL without satisfactory options after $\geq 2$ lines of systemic therapy. The reported ORR in the $E Z H 2$-mutated cohort from the phase 2 trial was $69 \%$, including $13 \%$ rate of $\mathrm{CR}$, whereas in $E Z H 2$-wild type these rates were $35 \%$ and $4 \%$, respectively. ${ }^{111}$ Median PFS was 14 and 11 months, respectively. As a targeted agent with high specificity, tazemetostat has an excellent toxicity profile, with rare non-hematologic grade $3 / 4$ adverse events (none more frequent than 3\%). Grade 1/2 reactions are more common, including nausea (23\%), diarrhea (18\%), alopecia (17\%), cough (16\%), fatigue (15\%) and upper respiratory infections $(15 \%)$. Eligibility for tazemetostat can be determined using the FDA-approved single-gene EZH2 mutation test or using a larger next-generation sequencing panel. For eligible older patients with FL, tazemetostat offers a particularly high-value therapeutic option.

\section{Immunotherapy: Emerging Direction}

Given the ongoing lack of curative options, many new agents are being studied for the treatment of iBCL (Table 3). Immunotherapy approaches are especially promising, as they offer novel mechanisms of action with high rates of CR and limited toxicity that is suitable for application among older patients. Both FL and MZL are known to strongly interact with the immune microenvironment, providing an opportunity to treat them by way of inducing immune attack or through immunomodulation within the tumor stroma. ${ }^{112,113}$

\section{Antibody-Drug Conjugates}

Antibody-drug conjugates (ADC) combine a lymphomatargeting monoclonal antibody with a cytotoxic small molecule to be internalized into the malignant cell upon binding. The goal is to increase tumor killing with minimal systemic toxicity. The first ADC established in lymphoma space, brentuximab vedotin, is unsuitable for FL or MZL which do not express its target CD30. Recently, ADCs targeting CD79b, CD22, or CD19 have been investigated in iBCL. Inotuzumab ozogamicin (which targets CD22) has been trialed in relapsed/refractory iBCL in a phase 2 study. ${ }^{114}$ The ORR was high (67\%), but unfortunately so were the rates of adverse events, and $58 \%$ of patients discontinued therapy secondary to toxicity.

Polatuzumab vedotin is an ADC with a CD79b target conjugated with monomethyl auristatin $\mathrm{E}$ (MMAE), a microtubule inhibitor. It is FDA-approved in combination with BR for DLBCL and has been studied in other relapsed/refractory lymphomas. ${ }^{115,116}$ The phase 2 ROMULUS study compared the combination of rituximab with polatuzumab or with pinatuzumab vedotin (a CD22: MMAE conjugate) among patients with relapsed/refractory DLBCL and FL. ${ }^{117}$ Among 20 patients with FL receiving rituximab and polatuzumab vedotin, $70 \%$ achieved a response, including $45 \%$ with a $\mathrm{CR}$, and 
median PFS was 15 months. Grade $\geq 3$ adverse events in FL were uncommon, including neutropenia (15\%), diarrhea $(10 \%)$ and dyspnea (5\%). Polatuzumab vedotin is associated with cumulative peripheral neuropathy, ultimately leading to treatment discontinuation in $55 \%$ of patients with FL. Although more research on this agent is needed in iBCL to determine optimal dose and treatment duration, high efficacy and low toxicity make this drug potentially appropriate for older patients.

Loncastuximab tesirine is a novel anti-CD19 ADC investigated for aggressive and indolent lymphomas. Current experience is limited to a Phase 1 trial which enrolled 14 subjects with FL. ${ }^{118}$ The maximum tolerated dose was not reached, and ORR in FL was 79\% with median duration of response not reached. The "naked" anti-CD19 antibody tafasitamab had a lower ORR in a phase 2a trial (29\% in FL), but it may synergize with lenalidomide as evidenced by its activity in DLBCL, which led to an FDA approval. ${ }^{119,120}$

\section{Chimeric Antigen Receptor (CAR) T Cell Therapy}

Autologous CD19-directed CAR-T cells, while initially studied in B-lymphoblastic leukemia and DLBCL, appear to work quite well in iBCL may be even better tolerated than in the aggressive histologies. ${ }^{121}$ The ZUMA-5 phase 2 trial of axicabtagene ciloleucel included patients with FL and MZL who had received $\geq 3$ lines of therapy, had performance status 0 or 1 and age up to $79 .{ }^{122}$ ORR was $92 \%(94 \%$ in $\mathrm{FL}$ and $85 \%$ in MZL) with a $76 \%$ CR rate ( $80 \%$ and $60 \%$, respectively) and projected 12 -month duration of response of $72 \%$. The hallmark risks of CAR

Table 3 Selected Emerging Therapies in FL and MZL

\begin{tabular}{|c|c|c|c|c|c|c|c|}
\hline Treatment & $\mathbf{N}$ & $\begin{array}{l}\text { Age, Median } \\
\text { (Range) }\end{array}$ & $\begin{array}{l}\text { Prior Lines, Median } \\
\text { (Range) }\end{array}$ & $\begin{array}{l}\text { Gr. } 3 / 4 \text { Adverse } \\
\text { Events }\end{array}$ & ORR & CR & $\begin{array}{l}\text { Median } \\
\text { PFS } \\
\text { (mo) }\end{array}$ \\
\hline $\begin{array}{l}\text { Polatuzumab vedotin } \\
+\mathrm{R}^{117}\end{array}$ & $20(\mathrm{FL})$ & 67 (59-74) & $2(2-4)$ & $50 \%$ & $70 \%^{a}$ & $45 \%^{a}$ & 15 \\
\hline $\begin{array}{l}\text { Loncastuximab } \\
\text { tesirine }{ }^{1 / 8}\end{array}$ & $14(\mathrm{FL})$ & $63(20-87)^{b}$ & $3(1-13)$ & $77 \%$ & $79 \%^{a}$ & $64 \%^{a}$ & NR \\
\hline Tafasitamab 119 & $34(\mathrm{FL})$ & $62(40-87)$ & $3(1->3)$ & $27 \%$ & $29 \%$ & $9 \%$ & 9 \\
\hline $\begin{array}{l}\text { Axicabtagene } \\
\text { ciloleucel }^{122}\end{array}$ & $\begin{array}{l}124 \\
(F L) \\
22 \\
\text { (MZL) }\end{array}$ & 61 (34-79) & $3(1-10)$ & $86 \%$ & $\begin{array}{l}\text { FL } 94 \% \\
\text { MZL } \\
85 \%\end{array}$ & $\begin{array}{l}\text { FL } 80 \% \\
\text { MZL } \\
60 \%\end{array}$ & NR \\
\hline Blinatumomab $^{124}$ & $38(\mathrm{FL})$ & $65(20-80)^{b}$ & $3(1-10)$ & $90 \%$ & $80 \%^{a}$ & $40 \%^{a}$ & NR \\
\hline Mosunetuzumab $^{127}$ & $62(\mathrm{FL})$ & 59 (27-85) & $3(2-11)$ & $35 \%$ & $68 \%$ & $50 \%$ & 12 \\
\hline Epcoritamab $^{129}$ & $12(\mathrm{FL})$ & $73(35-84)$ & $4.5(1-18)$ & NR & $100 \%$ & $25 \%$ & NR \\
\hline Nivolumab $^{133}$ & $92(\mathrm{FL})$ & 67 (37-87) & $3(2-10)$ & $15 \%$ & $4 \%$ & $1 \%$ & 2 \\
\hline $\begin{array}{l}\text { Atezolizumab + Obi } \\
\operatorname{orR}^{|4|}\end{array}$ & $\begin{array}{l}21 \\
(\mathrm{MCL})\end{array}$ & $67(47-87)^{b}$ & $2(I-7)$ & $48 \%$ & $43 \%$ & $14 \%$ & NR \\
\hline $\begin{array}{l}\text { Avadomide + } \\
\text { Obi }^{140,142}\end{array}$ & $53(\mathrm{FL})$ & $61(26-83)^{b}$ & $3(2-4)$ & $46 \%$ & $76 \%^{a}$ & $47 \%^{a}$ & 17 \\
\hline Venetoclax ${ }^{137}$ & $29(\mathrm{FL})$ & $64(46-75)$ & $3(1-10)$ & $56 \%$ & $38 \%$ & $14 \%$ & 11 \\
\hline Venetoclax $+R^{138}$ & $52(\mathrm{FL})$ & $63(40-84)$ & $3(I-6)$ & $50 \%$ & $35 \%$ & $17 \%$ & 7 \\
\hline Zanubrutinib $^{143}$ & $\begin{array}{l}68 \\
(M Z L)\end{array}$ & 70 (37-95) & $2(I-6)$ & $29 \%$ & $60 \%$ & $15 \%$ & NR \\
\hline
\end{tabular}

Notes: ${ }^{\mathrm{a} F L}$ only; ${ }^{\mathrm{b}}$ for all histologies.

Abbreviations: AE, adverse event; BR, bendamustine and rituximab; CR, complete response; mo, months; NR, not reported; Obi, obinutuzumab; ORR, overall response rate; PFS, progression-free survival; $R$, rituximab. 
T-cell therapy, namely grade 3/4 cytokine release syndrome and neurotoxicity, were relatively reduced $(7 \%$ and $19 \%$, respectively) compared with observations from DLBCL. However, grade 3/4 adverse effects occurred overall in $86 \%$ of participants, including neutropenia in $33 \%$ and anemia in $23 \%$. Based on these results, axicabtagene ciloleucel has been approved by the FDA for treatment of FL. However, due to the need for lymphodepleting chemotherapy and the overall intensity of the procedure, CAR T-cell therapy, while highly effective and potentially curative, will likely remain an option for only few older/unfit patients with iBCL. Experience with CAR T-cells among older (age 65-76) patients with DLBCL is so far encouraging, with similar efficacy and rates of cytokine release syndrome, although higher neurotoxicity than among younger patients. ${ }^{123}$ However, the risk/benefit ratio of CAR T-cell therapy for $\mathrm{BBCL}$ in this population remains to be delineated.

\section{Bispecific Antibodies}

Bispecific antibodies are a novel class of immunotherapeutic agents which simultaneously bind an antigen (CD20 or CD19) on malignant B-cells, and CD3 on immune effector T-cells, thus facilitating the formation of the immune synapse and T-cell activation. They offer perhaps the most promising approach for relapsed/refractory and possibly also previously untreated iBCL. Their effects are similar to CAR T-cell therapy but without the need for lymphodepleting chemotherapy or apheresis. Their suitability for older and more frail patients who cannot tolerate cytotoxic chemotherapy has already been investigated in some early-phase trials. Blinatumomab (a CD19/CD3directed bispecific T-cell engager) was studied in a phase 1 trial for patients (up to age 80) with relapsed/refractory FL and other lymphomas. ${ }^{124}$ ORR among 15 patients with FL was $80 \%$ with $40 \%$ of patients attaining a $\mathrm{CR}$, but the use of blinatumomab is complicated by the need for continuous intravenous infusion over weeks as well as $22 \%$ rate of grade $\geq 3$ neurologic events.

Mosunetuzumab is a CD20/CD3 bispecific antibody which has been granted FDA breakthrough designation for FL. ${ }^{125}$ The phase 1 trial of mosunetuzumab in relapsed/refractory CD20-positive lymphomas included 62 patients with FL of whom $68 \%$ responded and $50 \%$ achieved a CR. ${ }^{126,127}$ So far, $74 \%$ of patients who attained $\mathrm{CR}$ have remained in remission, and median duration of response is 20 months. ${ }^{127}$ The average age of participants was 59 , but ranged up to 85 . One third of patients experienced a treatment-related grade $3 / 4$ adverse event, but they were mostly laboratory-based (neutropenia) and no grade $3 / 4$ cytokine release syndrome or neurotoxicity occurred. Of note, mosunetuzumab has been studied specifically in the population of older and unfit patients with DLBCL (median age of 84, range 67-100), demonstrating a similar toxicity profile. ${ }^{128}$ Due to its high efficacy and low toxicity, mosunetuzumab is moving forward with further testing in both relapsed/refractory and previously untreated FL/MZL. Other CD20/CD3 bispecific antibodies with ongoing studies in FL/MZL include epcoritamab, ${ }^{129}$ glofitamab, ${ }^{130}$ and odronextamab, but their safety in older patients will require more research. ${ }^{131}$

\section{Checkpoint Inhibitors}

Nivolumab as a single agent initially demonstrated promise with responses among 4 of $10 \mathrm{FL}$ patients in a phase 1 study. ${ }^{132}$ Unfortunately, in the subsequent phase 2 experience, the ORR was only $4 \%$ with no clear predictive biomarker. ${ }^{133}$ Single-agent PD-1 blockade may thus be ineffective in relapsed/refractory FL, although additional research aims to determine ways to enhance immune response or identify predictors of response. ${ }^{134,135}$ An intriguing case of a complete hematologic remission in SMZL after pembrolizumab has been reported, although otherwise data on checkpoint inhibitors in MZL are lacking. ${ }^{136}$

\section{Other Targeted Agents}

Even though the BCL2-IGH rearrangement constitutes a molecular hallmark of FL, single-agent BCL2 inhibitor venetoclax is less active in FL than in SLL/CLL or mantle cell lymphoma. Among 29 patients in a phase 1 trial of venetoclax (with dose escalated up to $1200 \mathrm{mg}$ ) ORR was $38 \%$ and $\mathrm{CR}$ rate was $14 \%$, with median PFS of 11 months. ${ }^{137}$ The combination of venetoclax $(800 \mathrm{mg}$ daily) with rituximab has also been compared against the triplet of BR plus venetoclax or against BR in a phase 2 study for relapsed/refractory FL. ${ }^{138}$ While the BR plus venetoclax demonstrated unacceptable toxicity, venetoclax with $\mathrm{R}$ had ORR of $35 \%$, CR rate of $17 \%$, and median PFS of 7 months, with a safety profile similar to that observed in CLL/SLL. ${ }^{138,139}$ These results were ultimately disappointing and indicate that further investigation of venetoclax in FL needs to identify patients who derive benefit from treatment with BCL2 inhibitors alone or in synergistic combinations. 
Given the activity of lenalidomide, novel immunomodulatory imide drugs (IMiDs) are also under investigation. Avadomide combined with obinutuzumab shows similar tolerability to R2 with a high (76\%) ORR in FL and $47 \%$ of patients achieving a CR. ${ }^{140}$ Other IMiDs/cereblon E3 ligase modulators like iberdomide (CC-220) and CC99282 are being further investigated to determine their activity in lymphoma.

\section{Conclusions}

First-line therapy for older patients with FL/MZL who are unfit for chemotherapy is still primarily based on rituximab monotherapy. Lenalidomide in combination with rituximab offers an alternative to chemotherapy with comparable efficacy, but toxicity is not sufficiently improved. In second and subsequent lines of treatment, novel therapies have changed the landscape, requiring a nuanced approach between FL and MZL subtypes, with many chemotherapy-free options possible for patients relapsing after anti-CD20 antibody therapy. The incoming wave of highly effective and relatively non-toxic immunotherapy approaches may further allow patients with $\mathrm{BCL}$ to manage their disease without the need for chemotherapy exposure in their lifetime.

\section{Disclosure}

Dr Adam J Olszewski reports research funding for the institution from Genentech, TG Therapeutics, and Acrotech Biopharma; grants from Adaptive Biotechnologies, during the conduct of the study. The authors report no other conflicts of interest in this work.

\section{References}

1. Surveillance, Epidemiology, and End Results (SEER) Program. SEER*Stat Database: incidence - SEER research limited-field data, 21 registries, Nov 2019 sub (2000-2017) - linked to county attributes - time dependent (1990-2017) income/ rurality,1969-2018 counties, National cancer institute, DCCPS, surveillance research program, released April 2020, based on the November 2019 submission. 2020. Available from: www.seer.cancer.gov. Accessed May 3, 2021 .

2. Olszewski AJ, Shafqat H, Ali S. Disparate survival outcomes after front-line chemoimmunotherapy in older patients with follicular, nodal marginal zone and small lymphocytic lymphoma. Leuk Lymphoma . 2015;56(4):942-950. doi:10.3109/10428194.20 14.936013

3. Swerdlow SH. WHO Classification of Tumours of Haematopoietic and Lymphoid Tissues. Revised 4th ed. Lyon: World Health Organization, International Agency for Research on Cancer; 2017.

4. Treon SP, Xu L, Guerrera ML, et al. Genomic landscape of waldenstrom macroglobulinemia and its impact on treatment strategies. J Clin Oncol. 2020;38(11):1198-1208. doi:10.1200/JCO.19.02314
5. Kahl BS, Yang DT. Follicular lymphoma: evolving therapeutic strategies. Blood. 2016;127(17):2055-2063. doi:10.1182/blood2015-11-624288

6. Bertoni F, Zucca E. State-of-the-art therapeutics: marginal-zone lymphoma. J Clin Oncol. 2005;23(26):6415-6420. doi:10.1200/ JCO.2005.05.018

7. Brady JL, Binkley MS, Hajj C, et al. Definitive radiotherapy for localized follicular lymphoma staged by (18)F-FDG PET-CT: a collaborative study by ILROG. Blood. 2019;133(3):237-245. doi:10.1182/blood-2018-04-843540

8. MacManus M, Fisher R, Roos D, et al. Randomized trial of systemic therapy after involved-field radiotherapy in patients with early-stage follicular lymphoma: TROG 99.03. J Clin Oncol. 2018;36(29):2918-2925. doi:10.1200/JCO.2018.77.9892

9. Iannitto E, Tripodo C. How I diagnose and treat splenic lymphomas. Blood. 2011;117(9):2585-2595. doi:10.1182/blood2010-09-271437

10. Zucca E, Bertoni F. The spectrum of MALT lymphoma at different sites: biological and therapeutic relevance. Blood. 2016;127 (17):2082-2092. doi:10.1182/blood-2015-12-624304

11. Ardeshna KM, Qian W, Smith P, et al. Rituximab versus a watch-and-wait approach in patients with advanced-stage, asymptomatic, non-bulky follicular lymphoma: an open-label randomised phase 3 trial. Lancet Oncol. 2014;15(4):424-435. doi:10.1016/S1470-2045(14)70027-0

12. Advani R, Rosenberg SA, Horning SJ. Stage I and II follicular non-Hodgkin's lymphoma: long-term follow-up of no initial therapy. J Clin Oncol. 2004;22(8):1454-1459. doi:10.1200/ JCO.2004.10.086

13. Rummel MJ, Niederle N, Maschmeyer G, et al. Bendamustine plus rituximab versus CHOP plus rituximab as first-line treatment for patients with indolent and mantle-cell lymphomas: an open-label, multicentre, randomised, phase 3 non-inferiority trial. Lancet. 2013;381(9873):1203-1210. doi:10.1016/S01406736(12)61763-2

14. Garcia Munoz R, Izquierdo-Gil A, Munoz A, Roldan-Galiacho V, Rabasa P, Panizo C. Lymphocyte recovery is impaired in patients with chronic lymphocytic leukemia and indolent non-Hodgkin lymphomas treated with bendamustine plus rituximab. Ann Hematol. 2014;93(11):1879-1887. doi:10.1007/s00277-0142135-8

15. Olszewski AJ, Reagan JL, Castillo JJ. Late infections and secondary malignancies after bendamustine/rituximab or RCHOP/ RCVP chemotherapy for B-cell lymphomas. Am J Hematol. 2018;93(1):E1-E3. doi:10.1002/ajh.24921

16. Fung M, Jacobsen E, Freedman A, et al. Increased risk of infectious complications in older patients with indolent non-hodgkin lymphoma exposed to bendamustine. Clin Infect Dis. 2019;68 (2):247-255. doi:10.1093/cid/ciy458

17. Mohile SG, Dale W, Somerfield MR, et al. Practical assessment and management of vulnerabilities in older patients receiving chemotherapy: ASCO guideline for geriatric oncology. J Clin Oncol. 2018;36(22):2326-2347. doi:10.1200/JCO.2018.78.8687

18. Abel GA, Klepin HD. Frailty and the management of hematologic malignancies. Blood. 2018;131(5):515-524. doi:10.1182/blood2017-09-746420

19. Rosko AE, Olin RL, Artz A, Wildes TM, Stauder R, Klepin HD A call to action in hematologic disorders: a report from the ASH scientific workshop on hematology and aging. J Geriatr Oncol. 2018;9(4):287-290. doi:10.1016/j.jgo.2018.04.006

20. Brice P, Bastion Y, Lepage E, et al. Comparison in low-tumorburden follicular lymphomas between an initial no-treatment policy, prednimustine, or interferon alfa: a randomized study from the Groupe d'Etude des Lymphomes Folliculaires. Groupe d'Etude des Lymphomes de l'Adulte. J Clin Oncol. 1997;15 (3):1110-1117. doi:10.1200/JCO.1997.15.3.1110 
21. Ardeshna KM, Smith P, Norton A, et al. Long-term effect of a watch and wait policy versus immediate systemic treatment for asymptomatic advanced-stage non-Hodgkin lymphoma: a randomised controlled trial. Lancet. 2003;362(9383):516-522. doi:10.1016/S0140-6736(03)14110-4

22. Solal-Celigny $\mathrm{P}$, Roy $\mathrm{P}$, Colombat $\mathrm{P}$, et al. Follicular lymphoma international prognostic index. Blood. 2004;104(5):1258-1265. doi:10.1182/blood-2003-12-4434

23. Thieblemont C, Cascione L, Conconi A, et al. A MALT lymphoma prognostic index. Blood. 2017;130(12):1409-1417. doi:10.1182/blood-2017-03-771915

24. Bachy E, Maurer MJ, Habermann TM, et al. A simplified scoring system in de novo follicular lymphoma treated initially with immunochemotherapy. Blood. 2018;132(1):49-58. doi:10.1182/ blood-2017-11-816405

25. Merli F, Luminari S, Tucci A, et al. Simplified geriatric assessment in older patients with diffuse large B-cell lymphoma: the prospective elderly project of the Fondazione Italiana Linfomi. J Clin Oncol. 2021;39(11):1214-1222. doi:10.1200/JCO.20.02465

26. Di M, Huntington SF, Olszewski AJ. Challenges and opportunities in the management of diffuse large B-cell lymphoma in older patients. Oncologist. 2021;26(2):120-132. doi:10.1002/onco.13610

27. Scheepers ERM, Vondeling AM, Thielen N, van der Griend R, Stauder R, Hamaker ME. Geriatric assessment in older patients with a hematologic malignancy: a systematic review. Haematologica. 2020;105(6):1484-1493. doi:10.3324/ haematol.2019.245803

28. Boccomini C, Ladetto M, Rigacci L, et al. A brief rituximab, bendamustine, mitoxantrone (R-BM) induction followed by rituximab consolidation in elderly patients with advanced follicular lymphoma: a Phase II study by the Fondazione Italiana Linfomi (FIL). Br J Haematol. 2021;193(2):280-289. doi:10.1111/ bjh. 17283

29. Liu MA, DuMontier C, Murillo A, et al. Gait speed, grip strength, and clinical outcomes in older patients with hematologic malignancies. Blood. 2019;134(4):374-382. doi:10.1182/ blood.2019000758

30. Hantel A, DuMontier C, Odejide OO, et al. Gait speed, survival, and recommended treatment intensity in older adults with blood cancer requiring treatment. Cancer. 2021;127(6):875-883. doi:10.1002/cncr.33344

31. Goede V, Bahlo J, Chataline V, et al. Evaluation of geriatric assessment in patients with chronic lymphocytic leukemia: results of the CLL9 trial of the German CLL study group. Leuk Lymphoma. 2016;57(4):789-796. doi:10.3109/ 10428194.2015.1091933

32. Colombat P, Salles G, Brousse N, et al. Rituximab (anti-CD20 monoclonal antibody) as single first-line therapy for patients with follicular lymphoma with a low tumor burden: clinical and molecular evaluation. Blood. 2001;97(1):101-106. doi:10.1182/blood. V97.1.101

33. Colombat P, Brousse N, Salles G, et al. Rituximab induction immunotherapy for first-line low-tumor-burden follicular lymphoma: survival analyses with 7-year follow-up. Ann Oncol. 2012;23(9):2380-2385. doi:10.1093/annonc/mds 177

34. Lockmer S, Ostenstad B, Hagberg H, et al. Chemotherapy-free initial treatment of advanced indolent lymphoma has durable effect with low toxicity: results from two nordic lymphoma group trials with more than 10 years of follow-up. J Clin Oncol. 2018:JCO1800262. doi:10.1200/JCO.18.00262

35. Kimby E, Ostenstad B, Brown P, et al. Two courses of four weekly infusions of rituximab with or without interferon-alpha2a: final results from a randomized Phase III study in symptomatic indolent B-cell lymphomas. Leuk Lymphoma. 2015;56(9):2598-2607. doi:10.3109/ 10428194.2015.1014363
36. Martinelli G, Schmitz SF, Utiger U, et al. Long-term follow-up of patients with follicular lymphoma receiving single-agent rituximab at two different schedules in trial SAKK 35/98. J Clin Oncol. 2010;28(29):4480-4484. doi:10.1200/JCO.2010.28.4786

37. Moccia AA, Taverna C, Schar S, et al. Prolonged rituximab maintenance in follicular lymphoma patients: long-term results of the SAKK 35/03 randomized trial. Blood Adv. 2020;4 (23):5951-5957. doi:10.1182/bloodadvances.2020002858

38. Kahl BS, Hong F, Williams ME, et al. Rituximab extended schedule or re-treatment trial for low-tumor burden follicular lymphoma: eastern cooperative oncology group protocol e4402. J Clin Oncol. 2014;32 (28):3096-3102. doi:10.1200/JCO.2014.56.5853

39. Williams ME, Hong F, Gascoyne RD, et al. Rituximab extended schedule or retreatment trial for low tumour burden non-follicular indolent B-cell non-Hodgkin lymphomas: Eastern cooperative oncology group protocol E4402. Br J Haematol. 2016;173 (6):867-875. doi:10.1111/bjh.14007

40. Wagner LI, Zhao F, Hong F, et al. Anxiety and health-related quality of life among patients with low-tumor burden non-Hodgkin lymphoma randomly assigned to two different rituximab dosing regimens: results from ECOG trial E4402 (RESORT). J Clin Oncol. 2015;33 (7):740-748. doi:10.1200/JCO.2014.57.6801

41. Davies A, Merli F, Mihaljević B, et al. Efficacy and safety of subcutaneous rituximab versus intravenous rituximab for first-line treatment of follicular lymphoma (SABRINA): a randomised, open-label, phase 3 trial. Lancet Haematol. 2017;4(6):e272e282. doi:10.1016/S2352-3026(17)30078-9

42. Ogura M, Sancho JM, Cho SG, et al. Efficacy, pharmacokinetics, and safety of the biosimilar CT-P10 in comparison with rituximab in patients with previously untreated low-tumour-burden follicular lymphoma: a randomised, double-blind, parallel-group, phase 3 trial. Lancet Haematol. 2018;5(11):e543-e553. doi:10.1016/ S2352-3026(18)30157-1

43. Maloney DG, Ogura M, Fukuhara N, et al. A phase 3 randomized study (HOMER) of ofatumumab vs rituximab in iNHL relapsed after rituximab-containing therapy. Blood Adv. 2020;4 (16):3886-3893. doi:10.1182/bloodadvances.2020001942

44. Rosenbaum CA, Jung SH, Pitcher B, et al. Phase 2 multicentre study of single-agent ofatumumab in previously untreated follicular lymphoma: CALGB 50901 (Alliance). Br J Haematol. 2019;185(1):53-64. doi:10.1111/bjh. 15768

45. Freeman CL, Sehn LH. A tale of two antibodies: obinutuzumab versus rituximab. Br J Haematol. 2018;182(1):29-45. doi:10.1111/bjh.15232

46. Salles GA, Morschhauser F, Solal-Celigny P, et al. Obinutuzumab (GA101) in patients with relapsed/refractory indolent non-Hodgkin lymphoma: results from the phase II GAUGUIN study. J Clin Oncol. 2013;31(23):2920-2926. doi:10.1200/ JCO.2012.46.9718

47. Sehn LH, Goy A, Offner FC, et al. Randomized phase II trial comparing obinutuzumab (GA101) with rituximab in patients with relapsed $\mathrm{CD} 20+$ indolent B-cell non-hodgkin lymphoma: final analysis of the GAUSS study. J Clin Oncol. 2015;33 (30):3467-3474. doi:10.1200/JCO.2014.59.2139

48. Marcus R, Davies A, Ando K, et al. Obinutuzumab for the first-line treatment of follicular lymphoma. $N$ Engl J Med. 2017;377(14):1331-1344. doi:10.1056/NEJMoa1614598

49. Sehn LH, Chua N, Mayer J, et al. Obinutuzumab plus bendamustine versus bendamustine monotherapy in patients with rituximab-refractory indolent non-Hodgkin lymphoma (GADOLIN): a randomised, controlled, open-label, multicentre, phase 3 trial. Lancet Oncol. 2016;17(8):1081-1093. doi:10.1016/ S1470-2045(16)30097-3

50. Fowler NH, Davis RE, Rawal S, et al. Safety and activity of lenalidomide and rituximab in untreated indolent lymphoma: an open-label, phase 2 trial. Lancet Oncol. 2014;15(12):1311-1318. doi:10.1016/S1470-2045(14)70455-3 
51. Martin P, Jung SH, Pitcher B, et al. A phase II trial of lenalidomide plus rituximab in previously untreated follicular non-Hodgkin's lymphoma (NHL): CALGB 50803 (Alliance). Ann Oncol. 2017;28(11):2806-2812. doi:10.1093/annonc/ $\operatorname{mdx} 496$

52. Morschhauser F, Fowler NH, Feugier P, et al. Rituximab plus lenalidomide in advanced untreated follicular lymphoma. $N$ Engl $J$ Med. 2018;379(10):934-947. doi:10.1056/ NEJMoa1805104

53. Thanarajasingam G, Leonard JP, Witzig TE, et al. Longitudinal toxicity over time (ToxT) analysis to evaluate tolerability: a case study of lenalidomide in the CALGB 50401 (Alliance) trial. Lancet Haematol. 2020;7(6):e490-e497. doi:10.1016/S2352-3026(20)30067-3

54. Ujjani CS, Jung SH, Pitcher B, et al. Phase 1 trial of rituximab, lenalidomide, and ibrutinib in previously untreated follicular lymphoma: alliance A051103. Blood. 2016;128(21):2510-2516. doi:10.1182/blood-2016-06-718106

55. Smith SM, Pitcher BN, Jung SH, et al. Safety and tolerability of idelalisib, lenalidomide, and rituximab in relapsed and refractory lymphoma: the alliance for clinical trials in oncology A051201 and A051202 phase 1 trials. Lancet Haematol. 2017;4(4):e176e182. doi:10.1016/S2352-3026(17)30028-5

56. Becnel MR, Nastoupil LJ, Samaniego F, et al. Lenalidomide plus rituximab $(\mathrm{R}(2))$ in previously untreated marginal zone lymphoma: subgroup analysis and long-term follow-up of an open-label phase 2 trial. Br J Haematol. 2019;185(5):874-882. doi:10.1111/bjh. 15843

57. Zucca E, Rondeau S, Vanazzi A, et al. Short regimen of rituximab plus lenalidomide in follicular lymphoma patients in need of first-line therapy. Blood. 2019;134(4):353-362. doi:10.1182/ blood-2018-10-879643

58. Morschhauser F, Radford J, Van Hoof A, et al. 90Yttriumibritumomab tiuxetan consolidation of first remission in advanced-stage follicular non-Hodgkin lymphoma: updated results after a median follow-up of 7.3 years from the international, randomized, phase III first-lineindolent trial. J Clin Oncol. 2013;31(16):1977-1983. doi:10.1200/JCO.2012.45.6400

59. Zinzani PL, Tani M, Pulsoni A, et al. Fludarabine and mitoxantrone followed by yttrium-90 ibritumomab tiuxetan in previously untreated patients with follicular non-Hodgkin lymphoma trial: a phase II non-randomised trial (FLUMIZ). Lancet Oncol. 2008;9 (4):352-358. doi:10.1016/S1470-2045(08)70039-1

60. Ibatici A, Pica GM, Nati S, et al. Safety and efficacy of (90) yttrium-ibritumomab-tiuxetan for untreated follicular lymphoma patients. An Italian cooperative study. Br J Haematol. 2014;164 (5):710-716. doi:10.1111/bjh.12695

61. Samaniego F, Berkova Z, Romaguera JE, et al. 90Y-ibritumomab tiuxetan radiotherapy as first-line therapy for early stage low-grade B-cell lymphomas, including bulky disease. $\mathrm{Br}$ J Haematol. 2014;167(2):207-213. doi:10.1111/bjh.13021

62. Scholz CW, Pinto A, Linkesch W, et al. (90)Yttrium-ibritumomab -tiuxetan as first-line treatment for follicular lymphoma: 30 months of follow-up data from an international multicenter phase II clinical trial. J Clin Oncol. 2013;31(3):308-313. doi:10.1200/JCO.2011.41.1553

63. Czuczman MS, Emmanouilides C, Darif M, et al. Treatmentrelated myelodysplastic syndrome and acute myelogenous leukemia in patients treated with ibritumomab tiuxetan radioimmunotherapy. J Clin Oncol. 2007;25(27):4285-4292. doi:10.1200/JCO.2006.09.2882

64. Epperla N, Pham AQ, Burnette BL, et al. Risk of histological transformation and therapy-related myelodysplasia/acute myeloid leukaemia in patients receiving radioimmunotherapy for follicular lymphoma. Br J Haematol. 2017;178(3):427-433. doi:10.1111/ bjh. 14688
65. Di M, Ollila TA, Olszewski AJ. Exposure to ibritumomab tiuxetan and incidence of treatment-related myeloid neoplasms among older patients with B-cell lymphoma: a population-based study. Leukemia. 2020;34(10):2794-2797. doi:10.1038/s41375-020-0798-9

66. Illidge TM, Mayes S, Pettengell R, et al. Fractionated (9)(0) Y-ibritumomab tiuxetan radioimmunotherapy as an initial therapy of follicular lymphoma: an international phase II study in patients requiring treatment according to GELF/BNLI criteria. $J$ Clin Oncol. 2014;32(3):212-218. doi:10.1200/JCO.2013.50.3110

67. Lossos IS, Fabregas JC, Koru-Sengul T, et al. Phase II study of (90)Y Ibritumomab tiuxetan (Zevalin) in patients with previously untreated marginal zone lymphoma. Leuk Lymphoma. 2015;56 (6):1750-1755. doi:10.3109/10428194.2014.975801

68. Dreyling M, Thieblemont C, Gallamini A, et al. ESMO Consensus conferences: guidelines on malignant lymphoma. part 2: marginal zone lymphoma, mantle cell lymphoma, peripheral T-cell lymphoma. Ann Oncol. 2013;24(4):857-877. doi:10.1093/annonc/ mds643

69. Zucca E, Conconi A, Martinelli G, et al. Final results of the IELSG-19 randomized trial of mucosa-associated lymphoid tissue lymphoma: improved event-free and progression-free survival with rituximab plus chlorambucil versus either chlorambucil or rituximab monotherapy. J Clin Oncol. 2017;35(17):1905-1912. doi:10.1200/JCO.2016.70.6994

70. Olszewski AJ, Ollila TA, Reagan JL. Bendamustine-rituximab does not improve survival over rituximab monotherapy for older patients with nodal or splenic marginal zone lymphoma. Blood. 2019;134(Supplement_1):2824. doi:10.1182/blood-2019-121841

71. Else M, Marin-Niebla A, de la Cruz F, et al. Rituximab, used alone or in combination, is superior to other treatment modalities in splenic marginal zone lymphoma. Br J Haematol. 2012;159 (3):322-328. doi:10.1111/bjh.12036

72. Hoskin P, Popova B, Schofield O, et al. 4 Gy versus 24 Gy radiotherapy for follicular and marginal zone lymphoma (FoRT): long-term follow-up of a multicentre, randomised, phase 3, non-inferiority trial. Lancet Oncol. 2021;22 (3):332-340. doi:10.1016/S1470-2045(20)30686-0

73. Kalpadakis C, Pangalis GA, Angelopoulou MK, et al. Treatment of splenic marginal zone lymphoma with rituximab monotherapy: progress report and comparison with splenectomy. Oncologist. 2013;18(2):190-197. doi:10.1634/theoncologist.2012-0251

74. Olszewski AJ, Ali S. Comparative outcomes of rituximab-based systemic therapy and splenectomy in splenic marginal zone lymphoma. Ann Hematol. 2014;93(3):449-458. doi:10.1007/s00277-013-1900-4

75. Kalpadakis C, Pangalis GA, Sachanas S, et al. Rituximab monotherapy in splenic marginal zone lymphoma: prolonged responses and potential benefit from maintenance. Blood. 2018;132 (6):666-670. doi:10.1182/blood-2018-02-833608

76. Hermine $\mathrm{O}$, Lefrère $\mathrm{F}$, Bronowicki JP, et al. Regression of splenic lymphoma with villous lymphocytes after treatment of hepatitis C virus infection. $N$ Engl $J$ Med. 2002;347(2):89-94. doi:10.1056/NEJMoa013376

77. Xing KH, Kahlon A, Skinnider BF, et al. Outcomes in splenic marginal zone lymphoma: analysis of 107 patients treated in British Columbia. $B r \quad J$ Haematol. 2015;169(4):520-527. doi:10.1111/bjh. 13320

78. El Weshi A, Ribrag V, Girinski T, El Hajj M, Dhermain F, Bayle C. Low and medium dose spleen radiation therapy are able to induce long-term responses in splenic lymphoma with villous lymphocytes. Br J Haematol. 1998;103(4):1212-1213. doi:10.1046/j.1365-2141.1998.1161f.x

79. Casulo C, Byrtek M, Dawson KL, et al. Early relapse of follicular lymphoma after rituximab plus cyclophosphamide, doxorubicin, vincristine, and prednisone defines patients at high risk for death: an analysis from the national lymphocare study. J Clin Oncol. 2015;33(23):2516-2522. doi:10.1200/JCO.2014.59.7534 
80. Moccia AA, Schar S, Hayoz S, et al. Prognostic value of POD24 validation in follicular lymphoma patients initially treated with chemotherapy-free regimens in a pooled analysis of three randomized trials of the Swiss group for clinical cancer research (SAKK). Br J Haematol. 2021;192(6):1031-1034. doi:10.1111/ bjh. 17045

81. Leblond V, Johnson S, Chevret S, et al. Results of a randomized trial of chlorambucil versus fludarabine for patients with untreated Waldenstrom macroglobulinemia, marginal zone lymphoma, or lymphoplasmacytic lymphoma. J Clin Oncol. 2013;31 (3):301-307. doi:10.1200/JCO.2012.44.7920

82. Zucca E, Conconi A, Laszlo D, et al. Addition of rituximab to chlorambucil produces superior event-free survival in the treatment of patients with extranodal marginal-zone B-cell lymphoma: 5-year analysis of the IELSG-19 randomized study. J Clin Oncol. 2013;31(5):565-572. doi:10.1200/JCO.2011.40.6272

83. Martinelli G, Laszlo D, Bertolini F, et al. Chlorambucil in combination with induction and maintenance rituximab is feasible and active in indolent non-Hodgkin's lymphoma. $\mathrm{Br} J$ Haematol. 2003;123(2):271-277. doi:10.1046/j.1365-2141.2003.04586.x

84. Davis TA, Grillo-López AJ, White CA, et al. Rituximab antiCD20 monoclonal antibody therapy in non-Hodgkin's lymphoma: safety and efficacy of re-treatment. J Clin Oncol. 2000;18 (17):3135-3143. doi:10.1200/JCO.2000.18.17.3135

85. Vanazzi A, Grana C, Crosta C, et al. Efficacy of (9)(0) Yttrium-ibritumomab tiuxetan in relapsed/refractory extranodal marginal-zone lymphoma. Hematol Oncol. 2014;32(1):10-15. doi:10.1002/hon.2078

86. Gordon LI, Molina A, Witzig T, et al. Durable responses after ibritumomab tiuxetan radioimmunotherapy for $\mathrm{CD} 20+\mathrm{B}$-cell lymphoma: long-term follow-up of a phase 1/2 study. Blood. 2004;103(12):4429-4431. doi:10.1182/blood-2003-11-3883

87. Emmanouilides C, Witzig TE, Gordon LI, et al. Treatment with yttrium 90 ibritumomab tiuxetan at early relapse is safe and effective in patients with previously treated B-cell nonHodgkin's lymphoma. Leuk Lymphoma. 2006;47(4):629-636. doi:10.1080/10428190500376076

88. Witzig TE, Flinn IW, Gordon LI, et al. Treatment with ibritumomab tiuxetan radioimmunotherapy in patients with rituximabrefractory follicular non-Hodgkin's lymphoma. J Clin Oncol. 2002;20(15):3262-3269. doi:10.1200/JCO.2002.11.017

89. Witzig TE, Gordon LI, Cabanillas F, et al. Randomized controlled trial of yttrium-90-labeled ibritumomab tiuxetan radioimmunotherapy versus rituximab immunotherapy for patients with relapsed or refractory low-grade, follicular, or transformed B-cell non-Hodgkin's lymphoma. J Clin Oncol. 2002;20 (10):2453-2463. doi:10.1200/JCO.2002.11.076

90. Lolli G, Argnani L, Broccoli A, et al. (90) Y-ibritumomab tiuxetan in patients with extra-nodal marginal zone B-cell lymphoma of mucosa-associated lymphoid tissue (MALT lymphoma) - The Zeno Study. Br J Haematol. 2020;189(1):e6-e9. doi:10.1111/ bjh. 16404

91. Leonard JP, Trneny M, Izutsu K, et al. AUGMENT: a phase III study of lenalidomide plus rituximab versus placebo plus rituximab in relapsed or refractory indolent lymphoma. J Clin Oncol. 2019;37(14):1188-1199. doi:10.1200/JCO.19.00010

92. Morschhauser F, Le Gouill S, Feugier P, et al. Obinutuzumab combined with lenalidomide for relapsed or refractory follicular B-cell lymphoma (GALEN): a multicentre, single-arm, phase 2 study. Lancet Haematol. 2019;6(8):e429-e437. doi:10.1016/ S2352-3026(19)30089-4

93. Leonard JP, Jung SH, Johnson J, et al. Randomized trial of lenalidomide alone versus lenalidomide plus rituximab in patients with recurrent follicular lymphoma: CALGB 50401 (Alliance). $J \quad$ Clin Oncol. 2015;33(31):3635-3640. doi:10.1200/ JCO.2014.59.9258
94. Witzig TE, Wiernik PH, Moore T, et al. Lenalidomide oral monotherapy produces durable responses in relapsed or refractory indolent non-Hodgkin's lymphoma. J Clin Oncol. 2009;27 (32):5404-5409. doi:10.1200/JCO.2008.21.1169

95. Witlox WJA, Grimm SE, Riemsma R, et al. Lenalidomide with rituximab for previously treated follicular lymphoma and marginal zone lymphoma: an evidence review group perspective of a NICE single technology appraisal. Pharmacoeconomics. 2021;39(2):171-180. doi:10.1007/s40273-020-00971-x

96. von Keudell G, Moskowitz AJ. The role of PI3K inhibition in lymphoid malignancies. Curr Hematol Malig Rep. 2019;14 (5):405-413. doi:10.1007/s11899-019-00540-w

97. Cheah CY, Fowler NH. Idelalisib in the management of lymphoma. Blood. 2016;128(3):331-336. doi:10.1182/blood2016-02-702761

98. Gopal AK, Kahl BS, de Vos S, et al. PI3Kdelta inhibition by idelalisib in patients with relapsed indolent lymphoma. $N$ Engl J Med. 2014;370(11):1008-1018. doi:10.1056/NEJMoa1314583

99. Bird ST, Tian F, Flowers N, et al. Idelalisib for treatment of relapsed follicular lymphoma and chronic lymphocytic leukemia: a comparison of treatment outcomes in clinical trial participants vs medicare beneficiaries. JAMA Oncol. 2020;6(2):248-254. doi:10.1001/jamaoncol.2019.3994

100. Coutre SE, Barrientos JC, Brown JR, et al. Management of adverse events associated with idelalisib treatment: expert panel opinion. Leuk Lymphoma. 2015;56(10):2779-2786. doi:10.3109/ 10428194.2015.1022770

101. Flinn IW, Miller CB, Ardeshna KM, et al. DYNAMO: a phase II study of duvelisib (IPI-145) in patients with refractory indolent non-Hodgkin lymphoma. J Clin Oncol. 2019;37(11):912-922. doi:10.1200/JCO.18.00915

102. Burris HA, Flinn IW, Patel MR, et al. Umbralisib, a novel PI3Kdelta and casein kinase-1epsilon inhibitor, in relapsed or refractory chronic lymphocytic leukaemia and lymphoma: an open-label, phase 1, dose-escalation, first-in-human study. Lancet Oncol. 2018;19(4):486-496. doi:10.1016/S1470-2045(18) 30082-2

103. Fowler NH, Samaniego F, Jurczak W, et al. Umbralisib, a dual $\mathrm{PI} 3 \mathrm{Kdelta} / \mathrm{CK} 1$ epsilon Inhibitor in patients with relapsed or refractory indolent lymphoma. J Clin Oncol. 2021:JCO2003433. doi: $10.1200 /$ JCO.20.03433

104. Dreyling M, Santoro A, Mollica L, et al. Phosphatidylinositol 3-kinase inhibition by copanlisib in relapsed or refractory indolent lymphoma. $J$ Clin Oncol. 2017;35(35):3898-3905. doi: 10.1200/JCO.2017.75.4648

105. Panayiotidis P, Follows GA, Mollica L, et al. Efficacy and safety of copanlisib in patients with relapsed or refractory marginal zone lymphoma. Blood Adv. 2021;5(3):823-828. doi:10.1182/ bloodadvances.2020002910

106. Gopal AK, Schuster SJ, Fowler NH, et al. Ibrutinib as treatment for patients with relapsed/refractory follicular lymphoma: results from the open-label, multicenter, phase II DAWN study. J Clin Oncol. 2018;36(23):2405-2412. doi:10.1200/JCO.2017.76. 8853

107. Bartlett NL, Costello BA, LaPlant BR, et al. Single-agent ibrutinib in relapsed or refractory follicular lymphoma: a phase 2 consortium trial. Blood. 2018;131(2):182-190. doi:10.1182/ blood-2017-09-804641

108. Noy A, de Vos S, Thieblemont C, et al. Targeting Bruton tyrosine kinase with ibrutinib in relapsed/refractory marginal zone lymphoma. Blood. 2017;129(16):2224-2232. doi:10.1182/blood2016-10-747345

109. Noy A, de Vos S, Coleman M, et al. Durable ibrutinib responses in relapsed/refractory marginal zone lymphoma: long-term follow-up and biomarker analysis. Blood Adv. 2020;4 (22):5773-5784. doi:10.1182/bloodadvances.2020003121 
110. Pastore A, Jurinovic V, Kridel R, et al. Integration of gene mutations in risk prognostication for patients receiving first-line immunochemotherapy for follicular lymphoma: a retrospective analysis of a prospective clinical trial and validation in a population-based registry. Lancet Oncol. 2015;16(9):1111-1122. doi:10.1016/ S1470-2045(15)00169-2

111. Morschhauser F, Tilly H, Chaidos A, et al. Tazemetostat for patients with relapsed or refractory follicular lymphoma: an open-label, single-arm, multicentre, phase 2 trial. Lancet Oncol. 2020;21(11):1433-1442. doi:10.1016/S1470-2045(20)30441-1

112. Dave SS, Wright G, Tan B, et al. Prediction of survival in follicular lymphoma based on molecular features of tumor-infiltrating immune cells. $N$ Engl J Med. 2004;351 (21):2159-2169. doi:10.1056/NEJMoa041869

113. Fowler NH, Cheah CY, Gascoyne RD, et al. Role of the tumor microenvironment in mature B-cell lymphoid malignancies. Haematologica. 2016;101(5):531-540. doi:10.3324/haematol. 2015.139493

114. Goy A, Forero A, Wagner-Johnston N, et al. A phase 2 study of inotuzumab ozogamicin in patients with indolent B-cell non-Hodgkin lymphoma refractory to rituximab alone, rituximab and chemotherapy, or radioimmunotherapy. $\mathrm{Br} J$ Haematol. 2016;174(4):571-581. doi:10.1111/bjh.14094

115. Diefenbach C, Kahl BS, Banerjee L, et al. Polatuzumab vedotin plus obinutuzumab and lenalidomide in patients with relapsed/refractory follicular lymphoma: primary analysis of the full efficacy population in a phase Ib/II trial. Blood. 2019;134(Supplement_1):126. doi:10.1182/blood-2019-123 669

116. Palanca-Wessels MC, Czuczman M, Salles G, et al. Safety and activity of the anti-CD79B antibody-drug conjugate polatuzumab vedotin in relapsed or refractory B-cell non-Hodgkin lymphoma and chronic lymphocytic leukaemia: a phase 1 study. Lancet Oncol. 2015;16 (6):704-715. doi:10.1016/S1470-2045(15)70128-2

117. Morschhauser F, Flinn IW, Advani R, et al. Polatuzumab vedotin or pinatuzumab vedotin plus rituximab in patients with relapsed or refractory non-Hodgkin lymphoma: final results from a phase 2 randomised study (ROMULUS). Lancet Haematol. 2019;6(5): e254-e265. doi:10.1016/S2352-3026(19)30026-2

118. Hamadani M, Radford J, Carlo-Stella C, et al. Final results of a phase 1 study of loncastuximab tesirine in relapsed/refractory B-cell non-Hodgkin lymphoma. Blood. 2020. doi:10.1182/ blood.2020007512

119. Jurczak W, Zinzani PL, Gaidano G, et al. Phase IIa study of the CD19 antibody MOR208 in patients with relapsed or refractory B-cell non-Hodgkin's lymphoma. Ann Oncol. 2018;29 (5):1266-1272. doi:10.1093/annonc/mdy056

120. Salles G, Duell J, Gonzalez Barca E, et al. Tafasitamab plus lenalidomide in relapsed or refractory diffuse large B-cell lymphoma (L-MIND): a multicentre, prospective, single-arm, phase 2 study. Lancet Oncol. 2020;21(7):978-988. doi:10.1016/S1470-2045(20) 30225-4

121. Jacobson CA, Maus MV. C(h)AR-ting a new course in incurable lymphomas: CAR $\mathrm{T}$ cells for mantle cell and follicular lymphomas. Blood Adv. 2020;4(22):5858-5862. doi:10.1182/ bloodadvances.2020003391

122. Jacobson C, Chavez JC, Sehgal AR, et al. Primary analysis of Zuma-5: a phase 2 study of Axicabtagene Ciloleucel (Axi-Cel) in patients with relapsed/refractory $(\mathrm{R} / \mathrm{R})$ indolent non-Hodgkin lymphoma (iNHL). Blood. 2020;136(Supplement 1): 40-41.

123. Neelapu SS, Jacobson CA, Oluwole OO, et al. Outcomes of older patients in ZUMA-1, a pivotal study of axicabtagene ciloleucel in refractory large B-cell lymphoma. Blood. 2020;135 (23):2106-2109. doi:10.1182/blood.2019004162
124. Goebeler ME, Knop S, Viardot A, et al. Bispecific T-cell engager (BiTE) antibody construct blinatumomab for the treatment of patients with relapsed/refractory non-Hodgkin lymphoma: final results from a phase I study. J Clin Oncol. 2016;34 (10):1104-1111. doi:10.1200/JCO.2014.59.1586

125. Sun LL, Ellerman D, Mathieu M, et al. Anti-CD20/CD3 T cell-dependent bispecific antibody for the treatment of B cell malignancies. Sci Transl Med. 2015;7(287):287ra270. doi:10.1126/scitranslmed.aaa4802

126. Budde LE, O’Hear C, Li CC, et al. Mosunetuzumab induces complete remissions in poor prognosis non-Hodgkin lymphoma patients, including those who are resistant to or relapsing after chimeric antigen receptor T-cell (CAR-T) therapies, and is active in treatment through multiple lines. Blood. 2019;134 (Supplement_1):6. doi:10.1182/blood-2019-123742

127. Assouline SE, Kim WS, Sehn LH, et al. Mosunetuzumab shows promising efficacy in patients with multiply relapsed follicular lymphoma: updated clinical experience from a phase I dose-escalation trial. Blood. 2020;136(Supplement 1):42-44. doi:10.1182/blood-2020-135839

128. Olszewski AJ, Avigdor A, Babu S, et al. Single-agent mosunetuzumab is a promising safe and efficacious chemotherapy-free regimen for elderly/unfit patients with previously untreated diffuse large B-cell lymphoma. Blood. 2020;136(Supplement 1):43-45. doi:10.1182/blood-2020-136255

129. Hutchings M, Mous R, Clausen MR, et al. Subcutaneous epcoritamab induces complete responses with an encouraging safety profile across relapsed/refractory B-cell non-Hodgkin lymphoma subtypes, including patients with prior CAR-T therapy: updated dose escalation data. Blood. 2020;136(Supplement 1):45-46. doi:10.1182/blood-2020-133820

130. Hutchings M, Morschhauser F, Iacoboni G, et al. Glofitamab, a novel, bivalent CD20-targeting T-cell-engaging bispecific antibody, induces durable complete remissions in relapsed or refractory B-cell lymphoma: a phase I trial. J Clin Oncol. 2021: JCO2003175. doi:10.1200/JCO.20.03175

131. Bannerji R, Allan JN, Arnason JE, et al. Odronextamab (REGN1979), a human CD20 x CD3 bispecific antibody, induces durable, complete responses in patients with highly refractory B-cell non-Hodgkin lymphoma, including patients refractory to CAR T therapy. Blood. 2020;136(Supplement 1):42-43. doi:10.1182/blood-2020-136659

132. Lesokhin AM, Ansell SM, Armand P, et al. Nivolumab in patients with relapsed or refractory hematologic malignancy: preliminary results of a phase Ib study. J Clin Oncol. 2016;34(23):2698-2704. doi:10.1200/JCO.2015.65.9789

133. Armand P, Janssens A, Gritti G, et al. Efficacy and safety results from CheckMate 140, a phase 2 study of nivolumab for relapsed/ refractory follicular lymphoma. Blood. 2021;137(5):637-645. doi:10.1182/blood.2019004753

134. Yang ZZ, Kim HJ, Wu H, et al. TIGIT expression is associated with T-cell suppression and exhaustion and predicts clinical outcome and anti-PD-1 response in follicular lymphoma. Clin Cancer Res. 2020;26(19):5217-5231. doi:10.1158/1078-0432.CCR-20-0558

135. Herrera AF, Goy A, Mehta A, et al. Safety and activity of ibrutinib in combination with durvalumab in patients with relapsed or refractory follicular lymphoma or diffuse large B-cell lymphoma. Am J Hematol. 2020;95(1):18-27. doi:10.1002/ajh.25659

136. Miller PG, Sperling AS, Gibson CJ, et al. A deep molecular response of splenic marginal zone lymphoma to front-line checkpoint blockade. Haematologica. 2021;106(2):651-654. doi:10.3324/haematol.2020.258426

137. Davids MS, Roberts AW, Seymour JF, et al. Phase I first-inhuman study of venetoclax in patients with relapsed or refractory non-Hodgkin lymphoma. J Clin Oncol. 2017;35(8):826-833. doi:10.1200/JCO.2016.70.4320 
138. Zinzani PL, Flinn IW, Yuen SLS, et al. Venetoclax-rituximab with or without bendamustine vs bendamustine-rituximab in relapsed/ refractory follicular lymphoma. Blood. 2020;136(23):2628-2637. doi:10.1182/blood.2020005588

139. Seymour JF, Kipps TJ, Eichhorst B, et al. Venetoclax-rituximab in relapsed or refractory chronic lymphocytic leukemia. $N$ Engl J Med. 2018;378(12):1107-1120. doi:10.1056/NEJMoa1713976

140. Michot JM, Bouabdallah R, Vitolo U, et al. Avadomide plus obinutuzumab in patients with relapsed or refractory B-cell non-Hodgkin lymphoma (CC-122-NHL-001): a multicentre, dose escalation and expansion phase 1 study. Lancet Haematol. 2020;7(9):e649-e659. doi:10.1016/S2352-3026(20)30208-8

141. Panayiotidis P, Tumyan G, Thieblemont C, et al. Primary Analysis of the Tegar Study: a Phase II Study Exploring the Safety and Efficacy of Atezolizumab in Combination with Obinutuzumab or Rituximab in Patients with Relapsed or Refractory (R/R) Mantle Cell Lymphoma (MCL), Marginal Zone Lymphoma (MZL), or Waldenstrom Macroglobulinemia (WM). Blood. 2020;136(Supplement 1):2-3.
142. Michot JM, Bouabdallah R, Doorduijn JK, et al. Long-term results from a phase $1 \mathrm{~b}$ study of avadomide in combination with obinutuzumab in patients with relapsed and/or refractory B-cell non-Hodgkin lymphoma. Blood. 2020;136(Supplement 1):41-42. doi:10.1182/blood-2020-134949

143. Opat S, Tedeschi A, Linton K, et al. Efficacy and safety of zanubrutinib in patients with relapsed/refractory marginal zone lymphoma: initial results of the MAGNOLIA (BGB-3111-214) trial. Blood. 2020;136(Supplement 1):28-30. doi:10.1182/blood2020-134611

\section{Publish your work in this journal}

Cancer Management and Research is an international, peer-reviewed open access journal focusing on cancer research and the optimal use of preventative and integrated treatment interventions to achieve improved outcomes, enhanced survival and quality of life for the cancer patient.
The manuscript management system is completely online and includes a very quick and fair peer-review system, which is all easy to use. Visit http://www.dovepress.com/testimonials.php to read real quotes from published authors. 